\title{
Data Dependence, Strict Fixed Point Results, and Well-Posedness of Multivalued Weakly Picard Operators
}

\author{
Azhar Hussain $\mathbb{D}^{1},{ }^{1}$ Ahsan Ali, ${ }^{1}$ Vahid Parvaneh $\mathbb{D D}^{2}{ }^{2}$ and Hassen Aydi $\mathbb{D}^{3,4,5}$ \\ ${ }^{1}$ Department of Mathematics, University of Sargodha, Sargodha 40100, Pakistan \\ ${ }^{2}$ Department of Mathematics, Gilan-E-Gharb Branch, Islamic Azad University, Gilan-E-Gharb, Iran \\ ${ }^{3}$ Institut Supérieur d'Informatique et des Techniques de Communication, Université de Sousse, H. Sousse 4000, Tunisia \\ ${ }^{4}$ China Medical University Hospital, China Medical University, Taichung 40402, Taiwan \\ ${ }^{5}$ Department of Mathematics and Applied Mathematics, Sefako Makgatho Health Sciences University, \\ Ga-Rankuwa, South Africa
}

Correspondence should be addressed to Vahid Parvaneh; zam.dalahoo@gmail.com and Hassen Aydi; hassen.aydi@isima.rnu.tn Received 5 January 2021; Revised 31 January 2021; Accepted 1 March 2021; Published 16 March 2021

Academic Editor: Naeem Saleem

Copyright (c) 2021 Azhar Hussain et al. This is an open access article distributed under the Creative Commons Attribution License, which permits unrestricted use, distribution, and reproduction in any medium, provided the original work is properly cited.

In this paper, we introduce the notion of $(\mathfrak{g}, \mathfrak{r})$-contractive multivalued weakly Picard operators via simulation functions, named as $\mathscr{Z}_{(\mathfrak{g}, \mathfrak{r})}$-contractions. We present some related fixed point theorems. We investigate data dependence and strict fixed point results. The well-posedness for such operators is also considered. Moreover, we generalize the results of Moţ and Petruşel. To show the usability of our results, we give some examples and an application to resolve a functional equation arising in dynamical systems.

\section{Introduction and Preliminaries}

The fundamental theorem concerning the existence of a fixed point for a self-mapping on a metric space $(\mathcal{N}, d)$ is due to Banach [1].

Definition 1. A self-map $\mathbb{Q}$ on $\mathcal{N}$ is called a contraction if there exists $\widetilde{p} \in[0,1)$ such that

$$
d(\mathscr{Q} \mu, \mathbb{Q} \omega) \leq \widetilde{p} d(\mu, \omega)
$$

holds for all $\mu, \omega \in \mathcal{N}$.

On the basis of the above definition, Banach [1] in 1922 stated the following well-known contraction principle.

Theorem 1. Let $\mathbb{Q}: \mathcal{N} \longrightarrow \mathcal{N}$ be a contraction mapping defined on a complete metric space $(\mathcal{N}, d)$. Then, $\mathbb{Q}$ possesses a unique fixed point $\mu^{*}$ in $\mathcal{N}$. Furthermore, for any $\mu \in \mathcal{N}$, we have

$$
\lim _{\mathbf{n} \longrightarrow \infty} \mathbb{Q}^{\mathbf{n}} \mu=\mu^{*}
$$

with

$$
d\left(\mathscr{Q}^{\mathbf{n}} \mu, \mu^{*}\right) \leq \frac{\widetilde{p}^{\mathbf{n}}}{1-\widetilde{p}} d(\mu, Q \mu) .
$$

In 1969, investigating the case for multivalued mappings, Nadler [2] proved that the multivalued contraction mapping possesses at least one fixed point. Before moving towards this new generalization, we recall that $\mathfrak{P}(\mathcal{N}), \mathfrak{C}(\mathscr{N}), \mathscr{C} \mathscr{B}(\mathcal{N})$, and $\mathscr{K}(\mathcal{N})$ the nonempty, closed, closed and bounded, and compact subsets of a metric space $(\mathcal{N}, d)$, respectively. For $\omega \in \mathscr{N}$ and $\mathscr{U}, \mathscr{V} \in \mathscr{C} \mathscr{B}(\mathcal{N})$, consider

$$
\mathscr{D}(\mathscr{U}, \mathscr{V})=\inf \{d(\sigma, \lambda): \sigma \in \mathscr{U}, \lambda \in \mathscr{V}\},
$$

the Pompeiu-Hausdorff metric is defined by

$$
\mathscr{H}(\mathscr{U}, \mathscr{V}):=\max \left\{\sup _{u \in \mathscr{U}} \mathscr{D}(u, \mathscr{V}), \sup _{v \in \mathscr{V}} \mathscr{D}(v, \mathscr{U})\right\},
$$


where $\mathscr{D}(\omega, \mathscr{V})=\inf _{v \in \mathscr{V}} d(\omega, v)$.

Lemma 1 (see [2]). Suppose $\mathscr{U} \subseteq \mathcal{N}$ and $\xi>1$. Then, for $\mu \in \mathcal{N}$, there is $v \in \mathscr{V}$ so that $d(\mu, v) \leq \xi \mathscr{D}(\mu, \mathscr{V})$.

Theorem 2 (see [2]). Let $\mathbb{Q}: \mathcal{N} \longrightarrow \mathscr{C} \mathscr{B}(\mathscr{N})$ be a multivalued contraction mapping on a complete metric space $(\mathcal{N}, d)$. Then, $\mathcal{Q}$ possesses a fixed point.

It is obvious that if $(\mathcal{N}, d)$ is a complete metric space, then the pair $(\mathscr{C} \mathscr{B}(\mathscr{N}), \mathscr{H})$ and $(\mathfrak{C}(\mathscr{N}), \mathscr{H})$ is also complete (see e.g., [3-5]).

Definition 2 (see [6]). A mapping $\mathbb{Q}: \mathcal{N} \longrightarrow \mathscr{C} \mathscr{B}(\mathcal{N})$ is called a multivalued weakly Picard (MWP) operator if, for all $\mu \in \mathcal{N}$ and $\omega \in \mathbb{Q} \mu$, there exists a sequence $\left\{\mu_{\mathbf{n}}\right\}$ in $\mathcal{N}$ such that (i) $\mu_{0}=\mu, \mu_{1}=\omega$

(ii) $\mu_{\mathbf{n}+1} \in \mathscr{Q} \mu_{\mathbf{n}}$, for all $\mathbf{n} \geq 0$

(iii) $\left\{\mu_{\mathbf{n}}\right\}$ is convergent and its limit is a fixed point of $\mathbb{Q}$

Popescu [7] defined the notion of $(\mathfrak{g}, \mathfrak{r})$-contractive multivalued operators.

Definition 3 (see [7]). A multivalued operator Q: $\mathcal{N} \longrightarrow \mathscr{C} \mathscr{B}(\mathcal{N})$ on a complete metric space $(\mathcal{N}, d)$ is called $(\mathfrak{B}, \mathfrak{r})$-contractive if $\mathfrak{r} \in(0,1), \mathfrak{g} \geq \mathfrak{r}$, and $\mu, \omega \in \mathscr{N}$ such that

$\mathscr{D}(\omega, \mathbb{Q} \mu) \leq \mathfrak{B} d(\omega, \mu), \quad$ implies $\mathscr{H}(\mathscr{Q} \mu, \mathscr{Q} \omega) \leq r M_{\mathscr{T}}(\mu, \omega)$,

where

$$
M_{\mathscr{T}}(\mu, \omega)=\max \left\{d(\mu, \omega), \mathscr{D}(\mu, \mathbb{Q} \mu), \mathscr{D}(\omega, \mathscr{Q} \omega), \frac{\mathscr{D}(\mu, \mathscr{Q} \omega)+\mathscr{D}(\omega, \mathscr{Q} \mu)}{2}\right\} .
$$

Popescu [7] showed that $(\mathfrak{g}, \mathfrak{r})$-contractive multivalued operators are Picard, while in the single-valued case, it was shown that such operator possesses a unique fixed point. Later on, Kamran and Hussain [8] generalized the results of Popescu [7] to a weakly $(\mathfrak{g}, \mathfrak{x})$-contractive multivalued operator. The set of fixed points of the mapping $\mathbb{Q}$ is defined as $\operatorname{Fix}(\mathscr{Q}):=\{\mu \in \mathcal{N}: \mu \in \mathscr{Q} \mu\}$, while the set of strict fixed points is defined as $\operatorname{SFix}(\mathbb{Q}):=\{\mu \in \mathcal{N}:\{\mu\}=Q Q \mu\}$. It is clear that $\operatorname{SFix}(Q) \subseteq \operatorname{Fix}(Q)$.

Definition 4. (see $[9,10])$. Let $Y \in \mathfrak{P}(\mathcal{N})$, where $(\mathcal{N}, d)$ is a metric space, and $\mathbb{Q}: \mathcal{N} \longrightarrow \mathfrak{C}(\mathcal{N})$ be a multivalued operator. Then, the fixed point problem is well-posed for $\mathbb{Q}$ appropriate to $\mathscr{D}$ if

(i) $\operatorname{Fix}(\mathscr{Q})=\{\mu\}$

(ii) For a sequence $\left\{\mu_{\mathbf{n}}\right\}$ in $Y, \mathscr{D}\left(\mu_{\mathbf{n}}, \mathscr{Q} \mu_{\mathbf{n}}\right) \longrightarrow 0$ as $\mathbf{n} \longrightarrow \infty$; then, $d\left(\mu_{\mathbf{n}}, \mu\right) \longrightarrow 0$ as $\mathbf{n} \longrightarrow \infty$

Observe that a fixed point problem, which is well-posed for $\mathscr{Q}$ appropriate to $\mathscr{D}$, is also well-posed for $\mathbb{Q}$ appropriate to $\mathscr{H}$. Moţ and Petruşel in [11] proved the results of strict fixed point sets, well-posedness, and also data dependence of the fixed point sets.

An important class of functions was proposed by Khojasteh and Shukla [12] and was named as the set of simulation functions. Let $\xi:[0, \infty) \times[0, \infty) \longrightarrow \mathbb{R}$ verify the following conditions:

$$
\begin{aligned}
& \left(\xi_{0}\right) \xi(0,0)=0 . \\
& \left(\xi_{1}\right) \xi(\mu, \omega)<\omega-\mu, \text { for all } \mu, \omega>0 . \\
& \left(\xi_{2}\right) \text { If }\left\{\omega_{\mathbf{n}}\right\} \text { and }\left\{\mu_{\mathbf{n}}\right\} \text { are sequences in }(0, \infty) \text { such that } \\
& \lim _{\mathbf{n} \longrightarrow \infty} \omega_{\mathbf{n}}=\lim _{\mathbf{n} \longrightarrow \infty} \mu_{\mathbf{n}}>0, \text { then }
\end{aligned}
$$

$$
\lim _{\mathbf{n} \longrightarrow \infty} \sup \xi\left(\mu_{\mathbf{n}}, \omega_{\mathbf{n}}\right)<0 .
$$

Such $\xi$ is known as a simulation function proposed by Khojasteh et al. Utilizing such broad class of functions, they defined the notion of $\mathscr{Z}$-contractions. We denote such class of functions by $\Delta$.

Definition 5 (see [12]). A self-map $\mathscr{Q}$ on $\mathcal{N}$ is said to be $\mathscr{Z}$-contraction appropriate to $\xi$ if the inequality

$$
\xi(d(\mathbb{Q} \mu, \mathbb{Q} \omega), d(\mu, \omega)) \geq 0, \quad \forall \mu, \omega \in \mathcal{N},
$$

is fulfilled.

On the basis of contraction mappings defined above, they gave a version of the contraction principle, which generalizes and unifies several existing fixed point results in the literature.

After this work, studies involving simulation functions have been performed by various researchers (see [13-15] and references therein). Later on, Argoubi et al. [16] reshaped the notion of a simulation function by withdrawing the assertion $\left(\xi_{0}\right)$. We denote such class of functions by $\Lambda$.

Example 1 (see [16]). Let $\xi_{\lambda}:[0, \infty) \times[0, \infty) \longrightarrow \mathbb{R}$ be a function defined by

$$
\xi_{\lambda}(\omega, \mu)= \begin{cases}1, & \text { if }(\omega, \mu)=(0,0), \\ \lambda \mu-\omega, & \text { otherwise }\end{cases}
$$

where $\lambda \in(0,1)$. Then, $\xi_{\lambda} \in \Lambda$.

Later on, the assertion $\left(\zeta_{3}\right)$ of a simulation function was replaced with $\left(\zeta_{3}^{\prime}\right)$ by Roldán-López-de-Hierro et al. [17]. 
$\left(\zeta_{3}^{\prime}\right)$ If $\left\{\mu_{\mathbf{n}}\right\}$ and $\left\{\omega_{\mathbf{n}}\right\}$ are sequences in $(0, \infty)$ such that $\lim _{\mathbf{n} \longrightarrow \infty} \mu_{\mathbf{n}}=\lim _{\mathbf{n} \longrightarrow \infty} \omega_{\mathbf{n}}>0$ and $\mu_{\mathbf{n}}<\omega_{\mathbf{n}}$, then

$$
\lim _{\mathbf{n} \longrightarrow \infty} \sup \zeta\left(\mu_{\mathbf{n}}, \omega_{\mathbf{n}}\right)<0 .
$$

The class of simulation functions $\zeta$ fulfilling $\left(\zeta_{1}\right),\left(\zeta_{2}\right)$, and $\left(\zeta_{3}^{\prime}\right)$ is known as the class of simulation functions in the manner of Roldan - Lopez-de-Hierro, and we denote it by $\Sigma$. We need the following lemma.

Lemma 2 (see [18]). Let $(\mathcal{N}, d)$ be a metric space and let $\left\{\mu_{n}\right\}$ be a sequence in $X$ such that

$$
\lim _{\mathbf{n} \longrightarrow \infty} d\left(\mu_{n}, \mu_{n+1}\right)=0 .
$$

If $\left\{\mu_{n}\right\}$ is not a Cauchy sequence in $\mathcal{N}$, then there exist $\varepsilon>0$ and two sequences $\mu_{m(k)}$ and $\mu_{n(k)}$ of positive integers such that $\mu_{n(k)}>\mu_{m(k)}>k$ and the following sequences tend to $\varepsilon^{+}$when $k \longrightarrow \infty$ :

$$
\begin{aligned}
& d\left(\mu_{m(k)}, \mu_{n(k)}\right), d\left(\mu_{m(k)}, \mu_{n(k)+1}\right), d\left(\mu_{m(k)-1}, \mu_{n(k)}\right), \\
& d\left(\mu_{m(k)-1}, \mu_{n(k)+1}\right), d\left(\mu_{m(k)+1}, \mu_{n(k)+1}\right) .
\end{aligned}
$$

The purpose of this paper is to introduce the notion of weakly multivalued $\mathscr{Z}_{(\mathfrak{s}, \mathfrak{r})}$-contractions and to prove some fixed point results. We also discuss examples to illustrate and elaborate these new concepts. After that, we present data dependence, strict fixed point set, and well-posedness results. Following these ideas, we generalize the Moț and Petruşel result. Moreover, we present an application to functional equations arising in dynamical systems to show the usability of our results.

\section{Main Results}

We begin with the following definition.

Definition 6. Let $(\mathcal{N}, d)$ be a metric space. A mapping Q: $\mathcal{N} \longrightarrow \mathscr{C} \mathscr{B}(\mathcal{N})$ is called a weakly multivalued $\mathscr{Z}_{(\mathfrak{g}, \mathfrak{r})}$-contraction with respect to $\zeta$ if there are $\mathfrak{r} \in(0,1)$, $\mathfrak{g} \geq \mathfrak{r}$, and $\mu, \omega \in \mathscr{N}$ so that

$$
\mathscr{D}(\omega, \mathbb{Q} \mu) \leq \mathfrak{g} d(\omega, \mu)
$$

implies

$$
\zeta(\mathscr{H}(Q \mu, Q \mathcal{Q} \omega), \mathfrak{r} M(\mu, \omega)) \geq 0,
$$

where $\mathfrak{r} \in(0,1), \mathscr{L}=L \geq 0$, and

$$
\begin{aligned}
\mathscr{N}(\mu, \omega)= & \max \{d(\mu, \omega), \mathscr{D}(\mu, \mathscr{Q} \mu), \mathscr{D}(\omega, \mathscr{Q} \omega), \\
& \left.\frac{1}{2}(\mathscr{D}(\mu, \mathbb{Q} \omega)+\mathscr{D}(\omega, \mathscr{Q} \mu))\right\} \\
& +\mathscr{L} \min \{d(\mu, \omega), d(\omega, \mathbb{Q} \mu)\} .
\end{aligned}
$$

Example 2. Let $\mathcal{N}=[0,1]$ and $d(\mu, \omega)=|\mu-\omega|$. Define Q: $\mathcal{N} \longrightarrow \mathscr{C} \mathscr{B}(\mathcal{N})$ by

$$
Q \mu=\left[0, \frac{\mu+1}{\mu+3}\right] .
$$

Choose $\mathfrak{g}=0.8$. For $\mu, \omega \in \mathcal{N}, \mu \neq \omega$, we have

$$
\mathscr{D}(\mu, \mathcal{Q} \omega)<\mathfrak{g} d(\mu, \omega)
$$

which implies

$$
\zeta(\mathscr{H}(\mathbb{Q} \mu, \mathbb{Q} \omega), \mathfrak{r} M(\mu, \omega))=(\mathfrak{r} M(\mu, \omega))-\mathscr{H}(\mathbb{Q} \mu, \mathbb{Q} \omega)
$$$$
\geq 0, \quad \because L \geq 0, \mathfrak{x} \in(0,1) \text {. }
$$

Hence, $\mathscr{Q}$ is a weakly multivalued $\mathscr{Z}_{(0.8, \mathfrak{r})}$-contraction.

Theorem 3. Let $(\mathcal{N}, d)$ be a complete metric space and Q: $\mathcal{N} \longrightarrow \mathscr{C} \mathscr{B}(\mathcal{N})$ be a weakly multivalued $\mathscr{Z}_{(\mathfrak{B}, \mathfrak{r})}$-contraction. Then, $\mathbb{Q}$ is a MWP operator.

Proof. Let $\mu_{0} \in \mathcal{N}, \mu_{1} \in \mathbb{Q} \mu_{0}$, and $\mathfrak{t}$ be a real number such that $0<\mathfrak{r} \leq \mathfrak{t} \leq \mathfrak{g}<1$. Choose $\mu_{2} \in \mathscr{Q} \mu_{1}$. Then,

$$
\mathscr{D}\left(\mu_{2}, \mathscr{Q} \mu_{1}\right)=0 \leq \mathfrak{g} d\left(\mu_{2}, \mu_{1}\right) \text {. }
$$

From (15) and $\zeta_{1} \in \mathscr{Z}$, we have

$$
0 \leq \zeta\left(\mathscr{H}\left(\mathscr{Q} \mu_{1}, \mathscr{Q} \mu_{2}\right), \mathfrak{r} M\left(\mu_{1}, \mu_{2}\right)\right)<\mathfrak{r} M\left(\mu_{1}, \mu_{2}\right)-\mathscr{H}\left(\mathscr{Q} \mu_{1}, \mathscr{Q} \mu_{2}\right),
$$

which implies

$$
\begin{aligned}
& \mathscr{H}\left(\mathscr{Q} \mu_{1}, \mathscr{Q} \mu_{2}\right)<\mathfrak{r} M\left(\mu_{1}, \mu_{2}\right), \\
& \mathscr{D}\left(\mu_{2}, \mathscr{Q} \mu_{2}\right) \leq \mathscr{H}\left(\mathscr{Q} \mu_{1}, \mathscr{Q} \mu_{2}\right)<\mathfrak{r} M\left(\mu_{1}, \mu_{2}\right) .
\end{aligned}
$$

Hence,

$$
\begin{aligned}
\mathscr{D} & \left(\mu_{2}, \mathscr{Q} \mu_{2}\right)<\mathfrak{r} M\left(\mu_{1}, \mu_{2}\right) \\
= & \mathfrak{r} \max \left\{d\left(\mu_{1}, \mu_{2}\right), \mathscr{D}\left(\mu_{1}, \mathscr{Q} \mu_{1}\right), \mathscr{D}\left(\mu_{2}, \mathscr{Q} \mu_{2}\right),\right. \\
& \left.\frac{1}{2}\left(\mathscr{D}\left(\mu_{1}, \mathscr{Q} \mu_{2}\right)+\mathscr{D}\left(\mu_{2}, \mathscr{Q} \mu_{1}\right)\right)\right\} \\
& +L \min \left\{d\left(\mu_{1}, \mu_{2}\right), d\left(\mu_{2}, \mathscr{Q} \mu_{1}\right)\right\}
\end{aligned}
$$

implies

$$
d\left(\mu_{2}, \mu_{3}\right)<\mathfrak{r} d\left(\mu_{1}, \mu_{2}\right)+L \min \left\{d\left(\mu_{1}, \mu_{2}\right), 0\right\}=\mathfrak{r} d\left(\mu_{1}, \mu_{2}\right) .
$$

Similarly, we can get a sequence $\left\{\mu_{\mathbf{n}}\right\}$ in $\mathcal{N}$ such that $\left\{\mu_{\mathbf{n}+1}\right\} \in \mathbb{Q} \mu_{\mathbf{n}}$ and

$$
d\left(\mu_{\mathbf{n}+1}, \mu_{\mathbf{n}+2}\right)<\mathfrak{r} d\left(\mu_{\mathbf{n}}, \mu_{\mathbf{n}+1}\right)<\mathfrak{r}^{\mathbf{n}} d\left(\mu_{1}, \mu_{2}\right) \leq \mathfrak{t}^{\mathbf{n}} d\left(\mu_{1}, \mu_{2}\right) .
$$

Since $\mathbf{n} \longrightarrow \infty, d\left(\mu_{\mathbf{n}+1}, \mu_{\mathbf{n}+2}\right) \longrightarrow 0$, this shows that $\left\{\mu_{\mathbf{n}}\right\}$ is a Cauchy sequence. $\mathcal{N}$ is complete, so there exists $u \in \mathcal{N}$ such that

$$
\lim _{\mathbf{n} \longrightarrow \infty} \mu_{\mathbf{n}}=u
$$


We now show that there exists a subsequence $\left\{\mu_{\mathbf{n}(\mathfrak{m})}\right\}$ of $\left\{\mu_{\mathbf{n}}\right\}$ such that

$$
\mathscr{D}\left(u, Q \mu_{\mathbf{n}(\mathfrak{m})}\right) \leq \mathfrak{g} d\left(u, \mu_{\mathbf{n}(\mathfrak{m})}\right), \quad \forall \mathfrak{m} \in \mathbb{N} .
$$

On the contrary, we assume that there is a positive integer $N \in \mathbb{N}$ such that

$$
\mathscr{D}\left(u, Q \mu_{\mathbf{n}}\right)>\mathfrak{s} d\left(u, \mu_{\mathbf{n}}\right), \quad \forall \mathbf{n} \geq N .
$$

This implies

$$
d\left(u, \mu_{\mathbf{n}+1}\right)>\mathfrak{s} d\left(u, \mu_{\mathbf{n}}\right), \quad \forall \mathbf{n} \geq N .
$$

By induction, we obtain

$$
d\left(u, \mu_{\mathbf{n}+p}\right)>\mathfrak{G}^{p} d\left(u, \mu_{\mathbf{n}}\right), \quad \forall \mathbf{n} \geq N, p \geq 1 .
$$

Recall that

$$
\begin{aligned}
& d\left(\mu_{\mathbf{n}+p}, \mu_{\mathbf{n}}\right) \leq d\left(\mu_{\mathbf{n}}, \mu_{\mathbf{n}+1}\right)+d\left(\mu_{\mathbf{n}+1}, \mu_{\mathbf{n}+2}\right) \\
& \quad+\cdots+d\left(\mu_{\mathbf{n}+p-1}, \mu_{\mathbf{n}+p}\right) \\
& <d\left(\mu_{\mathbf{n}}, \mu_{\mathbf{n}+1}\right)\left(1+\mathfrak{t}+\mathfrak{t}^{2}+\mathfrak{t}^{3}+\cdots+\mathfrak{t}^{p-1}\right) \\
& \leq \frac{1-\mathfrak{t}^{p}}{1-\mathfrak{t}} d\left(\mu_{\mathbf{n}}, \mu_{\mathbf{n}+1}\right)<\frac{1}{1-\mathfrak{t}} d\left(\mu_{\mathbf{n}}, \mu_{\mathbf{n}+1}\right), \quad \forall \mathbf{n} \geq N, p \geq 1 .
\end{aligned}
$$

Taking $p \longrightarrow \infty$, we get that

$$
d\left(u, \mu_{\mathbf{n}}\right)<\frac{1}{1-\mathfrak{t}} d\left(\mu_{\mathbf{n}}, \mu_{\mathbf{n}+1}\right), \quad \forall \mathbf{n} \geq 1 .
$$

Thus, we have

$$
\begin{aligned}
d\left(u, \mu_{\mathbf{n}+p}\right) & <\frac{1}{1-\mathfrak{t}} d\left(\mu_{\mathbf{n}+p}, \mu_{\mathbf{n}+p+1}\right) \\
& <\frac{\mathbf{t}^{p}}{1-\mathfrak{t}} d\left(\mu_{\mathbf{n}}, \mu_{\mathbf{n}+1}\right), \quad \forall \mathbf{n} \geq N, p \geq 1 .
\end{aligned}
$$

From (30) and (33), one writes

$$
d\left(u, \mu_{\mathbf{n}}\right)<\frac{(\mathfrak{t} / \mathfrak{G})^{p}}{1-\mathfrak{t}} d\left(\mu_{\mathbf{n}}, \mu_{\mathbf{n}+1}\right), \quad \forall \mathbf{n} \geq N .
$$

By taking $p \longrightarrow \infty$, we have $d\left(u, \mu_{\mathbf{n}}\right)=0$, for all $\mathbf{n} \geq N$. It is a contradiction with respect to equation (30). Therefore, there is a subsequence $\left\{\mu_{\mathbf{n}(\mathfrak{m})}\right\}$ of $\left\{\mu_{\mathbf{n}}\right\}$ such that

$$
\mathscr{D}\left(u, Q \mathcal{Q} \mu_{\mathbf{n}(\mathfrak{m})}\right) \leq \mathfrak{g} d\left(u, \mu_{\mathbf{n}(\mathfrak{m})}\right), \quad \forall \mathfrak{m} \in \mathbb{N},
$$

which implies

$$
\zeta\left(\mathscr{H}\left(\mathcal{Q} u, Q \mu_{\mathbf{n}(\mathfrak{m})}\right), \mathfrak{r} M\left(u, \mu_{\mathbf{n}(\mathfrak{m})}\right)\right) \geq 0 .
$$

Now, from (36) and using $\left(\zeta_{1}\right)$ together with $\left(\zeta_{2}\right)$, we have

$$
\begin{aligned}
0 \leq & \lim _{\mathbf{n} \longrightarrow \infty} \sup \left[\zeta\left(\mathscr{H}\left(\mathscr{Q} u, \mathscr{Q} \mu_{\mathbf{n}(\mathfrak{m})}\right), \mathfrak{r} M\left(u, \mu_{\mathbf{n}(\mathfrak{m})}\right)\right)\right] \\
< & \lim _{\mathbf{n} \longrightarrow \infty} \sup \left[\mathfrak{r} M\left(u, \mu_{\mathbf{n}(\mathfrak{m})}\right)-\mathscr{H}\left(\mathscr{Q} u, \mathscr{Q} \mu_{\mathbf{n}(\mathfrak{m})}\right)\right] \\
= & \lim _{\mathbf{n} \longrightarrow \infty} \sup \left[\mathfrak{r} \max \left\{d\left(u, \mu_{\mathbf{n}(\mathfrak{m})}\right), \mathscr{D}(u, \mathscr{Q} u), \mathscr{D}\left(\mu_{\mathbf{n}(\mathfrak{m})}, \mathscr{Q} \mu_{\mathbf{n}(\mathfrak{m})}\right), \frac{1}{2}\left(\mathscr{D}\left(u, \mathscr{Q} \mu_{\mathbf{n}(\mathfrak{m})}\right)+\mathscr{D}\left(\mu_{\mathbf{n}(\mathfrak{m})}, \mathscr{Q} u\right)\right)\right\}\right. \\
& \left.+L \min \left\{d\left(u, \mu_{\mathbf{n}(\mathfrak{m})}\right), \mathscr{D}\left(\mu_{\mathbf{n}(\mathfrak{m})}, \mathscr{Q} u\right)\right\}-\max \left\{\mathscr{D}\left(\mathscr{Q} u, \mathscr{Q} \mu_{\mathbf{n}(\mathfrak{m})}\right), \mathscr{D}\left(\mathscr{Q} \mu_{\mathbf{n}(\mathfrak{m})}, \mathscr{Q} u\right)\right\}\right] \\
= & \lim _{\mathbf{n} \longrightarrow \infty} \sup \left[\mathfrak{r} \max \left\{d\left(u, \mu_{\mathbf{n}(\mathfrak{m})}\right), \mathscr{D}(u, \mathscr{Q} u), \mathscr{D}\left(\mu_{\mathbf{n}(\mathfrak{m})}, \mu_{\mathbf{n}(\mathfrak{m})+1}\right), \frac{1}{2}\left(\mathscr{D}\left(u, \mu_{\mathbf{n}(\mathfrak{m})+1}\right)+\mathscr{D}\left(\mu_{\mathbf{n}(\mathfrak{m})}, \mathscr{Q} u\right)\right)\right\}\right. \\
& \left.+L \min \left\{\mathscr{D}\left(u, \mu_{\mathbf{n}(\mathfrak{m})}\right), \mathscr{D}\left(\mu_{\mathbf{n}(\mathfrak{m})}, \mathscr{Q} u\right)\right\}-\max \left\{\mathscr{D}\left(\mathscr{Q} u, \mu_{\mathbf{n}(\mathfrak{m})+1}\right), \mathscr{D}\left(\mu_{\mathbf{n}(\mathfrak{m})+1}, \mathscr{Q} u\right)\right\}\right] \\
= & \sup \left[\mathfrak{r} \max \left\{d(u, u), \mathscr{D}(u, \mathscr{Q} u), \mathscr{D}(u, u), \frac{1}{2}(\mathscr{D}(u, u)+\mathscr{D}(u, \mathscr{Q} u))\right\}\right. \\
& +L \min \{d(u, u), \mathscr{D}(u, \mathscr{Q} u)\}-\max \{\mathscr{D}(\mathscr{Q} u, u), \mathscr{D}(u, \mathscr{Q} u)\}] \\
= & \sup \left[\mathfrak{r} \max \left\{\mathscr{D}(u, \mathscr{Q} u), \frac{\mathscr{D}(u, \mathscr{Q} u)}{2}\right\}+L \min \{0, \mathscr{D}(u, \mathscr{Q} u)\}-\max \{\mathscr{D}(\mathscr{Q} u, u), \mathscr{D}(u, \mathscr{Q} u)\}\right] \\
= & \sup [\mathfrak{r} d(u, \mathscr{Q} u)+0-\mathscr{D}(u, \mathscr{Q} u)] \\
= & (\mathfrak{r}-1) \mathscr{D}(u, \mathscr{Q} u), \\
&
\end{aligned}
$$

where $\mathfrak{x} \in(0,1)$. So,

$$
0 \leq(\mathfrak{r}-1) \mathscr{D}(u, \mathscr{Q} u)<0 .
$$


From this contradiction, we get $\mathscr{D}(u, \mathbb{Q} u)=0$, that is, $u \in \mathbb{Q} u$. Hence, $\mathbb{Q}$ is a MWP operator.

Example 3. Let $\mathcal{N}=\{1,2,3,4,5\}$ and $d(\mu, \omega)=|\mu-\omega|$. Define

$$
Q \mu= \begin{cases}\{5\}, & \text { if } \mu \in\{5\}, \\ \{2,3\}, & \text { if } \mu \in\{2,3\}, \\ \{1,4\}, & \text { otherwise. }\end{cases}
$$

Now, for $\mu, \omega \in \mathscr{N}$, values of $d$, $\mathscr{D}$, and $\mathscr{H}$ for all possible pair of points are given in Table 1.

By choosing $\mathfrak{g}=0.5$, we have

$$
\begin{aligned}
& \mathscr{D}(1, \mathbb{Q} 2)>\mathfrak{g d}(1,2), \mathscr{D}(3, \mathbb{Q} 5)>\mathfrak{s} d(3,5) \text {, } \\
& \mathscr{D}(1, \mathbb{Q} 5)>\mathfrak{s d}(1,5), \mathscr{D}(4, \mathbb{Q} 3)>\mathfrak{s} d(4,3) \text {, } \\
& \mathscr{D}(2, \mathbb{Q} 1)>\mathfrak{g} d(2,1), \mathscr{D}(4, \mathscr{Q} 5)>\mathfrak{s} d(4,5) \text {, } \\
& \mathscr{D}(2, \mathbb{Q} 5)>\mathfrak{g} d(2,5), \mathscr{D}(5, \mathscr{Q} 3)>\mathfrak{s} d(5,3) \text {, } \\
& \mathscr{D}(3, \mathscr{Q} 1)>\mathfrak{g d}(3,1), \mathscr{D}(5, \mathscr{Q} 4)>\mathfrak{s} d(5,4) \text {, } \\
& \mathscr{D}(3, Q 4)>\mathfrak{S d}(3,4) \text {. }
\end{aligned}
$$

Furthermore, for $\mathfrak{S}=0.5$, we also have

$$
\begin{aligned}
& \mathscr{D}(1, \mathscr{Q} 1)=\mathfrak{g} d(1,1), \mathscr{D}(5, \mathscr{Q} 5)=\mathfrak{g} d(5,5) \text {, } \\
& \mathscr{D}(1, \mathbb{Q} 3)=\mathfrak{s} d(1,3), \mathscr{D}(1, \mathbb{Q} 4)<\mathfrak{g d}(1,4) \text {, } \\
& \mathscr{D}(2, \mathbb{Q} 2)=\mathfrak{g} d(2,2), \mathscr{D}(2, \mathbb{Q} 3)<\mathfrak{g} d(4,5) \text {, } \\
& \mathscr{D}(3, \mathscr{Q} 3)=\mathfrak{g} d(3,3), \mathscr{D}(3, \mathscr{Q} 2)<\mathfrak{g} d(5,3) \text {, } \\
& \mathscr{D}(4, \mathbb{Q} 2)=\mathfrak{s} d(4,2), \mathscr{D}(4, \mathscr{Q} 1)<\mathfrak{g} d(5,4) \text {, } \\
& \mathscr{D}(4, \mathbb{Q} 4)=\mathfrak{g} d(4,4), \mathscr{D}(5, \mathbb{Q} 1)<\mathfrak{g} d(5,1) \text {. }
\end{aligned}
$$

$$
\begin{aligned}
& \zeta(\mathscr{H}(\mathcal{Q} 1, \mathcal{Q} 1), \mathfrak{r} M(1,1))=\mathfrak{r} M(1,1))-\mathscr{H}(\mathscr{Q} 1, \mathcal{Q} 1)=(0.4)(0)+(1)(0)-0=0, \\
& \zeta(\mathscr{H}(Q 1, Q 3), \mathfrak{r} M(1,3))=\mathfrak{r} M(1,3))-\mathscr{H}(Q 1, Q 3)=(0.4)(2)+(1)(1)-1>0, \\
& \zeta(\mathscr{H}(\mathbb{Q} 2, \mathbb{Q} 2), \mathfrak{r} M(2,2))=\mathfrak{r} M(2,2))-\mathscr{H}(\mathbb{Q} 2, \mathbb{Q} 2)=(0.4)(0)+(1)(0)-0=0, \\
& \zeta(\mathscr{H}(Q 3, Q 3), \mathfrak{r} M(3,3))=\mathfrak{r} M(3,3))-\mathscr{H}(Q 3, Q 3)=(0.4)(0)+(1)(0)-0=0, \\
& \zeta(\mathscr{H}(Q 4, Q 2), \mathfrak{r} M(4,2))=\mathfrak{r} M(4,2))-\mathscr{H}(Q 4, Q 2)=(0.4)(2)+(1)(1)-1>0, \\
& \zeta(\mathscr{H}(Q 4, Q 4), \mathfrak{r} M(4,4))=\mathfrak{r} M(4,4))-\mathscr{H}(\mathbb{Q} 4, \mathbb{Q} 4)=(0.4)(0)+(1)(0)-0=0, \\
& \zeta(\mathscr{H}(Q 5, Q 5), \mathfrak{r} M(5,5))=\mathfrak{r} M(5,5))-\mathscr{H}(Q 25, Q 25)=(0.4)(0)+(1)(0)-0=0, \\
& \zeta(\mathscr{H}(Q 1, Q 4), \mathfrak{r} M(1,4))=\mathfrak{r} M(1,4))-\mathscr{H}(Q 1, Q 4)=(0.4)(3)+(1)(0)-0>0, \\
& \zeta(\mathscr{H}(Q 2, Q 3), \mathfrak{r} M(2,3))=\mathfrak{r} M(2,3))-\mathscr{H}(\mathscr{Q} 2, \mathbb{Q} 3)=(0.4)(1)+(1)(0)-0>0, \\
& \zeta(\mathscr{H}(Q 3, Q 2), \mathfrak{r} M(3,2))=\mathfrak{r} M(3,2))-\mathscr{H}(Q 3, Q 2)=(0.4)(1)+(1)(0)-0>0 \text {, } \\
& \zeta(\mathscr{H}(Q 4, Q 1), \mathfrak{r} M(4,1))=\mathfrak{r} M(4,1))-\mathscr{H}(Q 4, Q 1)=(0.4)(3)+(1)(0)-0>0, \\
& \zeta(\mathscr{H}(Q 5, Q 1), \mathfrak{r} M(5,1))=\mathfrak{r} M(5,1))-\mathscr{H}(Q 25, Q 1)=(0.4)(1)+(1)(4)-4>0 .
\end{aligned}
$$


TABle 1: Values of $d, \mathscr{D}$, and $\mathscr{H}$.

\begin{tabular}{|c|c|c|c|c|}
\hline$(\mu, \omega)$ & $d(\mu, \omega)$ & $\mathscr{D}(\mu, Q)$ & $\mathscr{D}(\omega, Q \mathcal{Q} \mu)$ & $\mathscr{H}(Q), Q \omega)$ \\
\hline$(1,1)$ & 0 & 0 & 0 & 0 \\
\hline$(1,2)$ & 1 & 0 & 1 & 1 \\
\hline$(1,3)$ & 2 & 0 & 1 & 1 \\
\hline$(1,4)$ & 3 & 0 & 0 & 0 \\
\hline$(1,5)$ & 4 & 0 & 1 & 4 \\
\hline$(2,1)$ & 1 & 0 & 1 & 1 \\
\hline$(2,2)$ & 0 & 0 & 0 & 0 \\
\hline$(2,3)$ & 1 & 0 & 0 & 0 \\
\hline$(2,4)$ & 2 & 0 & 1 & 1 \\
\hline$(2,5)$ & 3 & 0 & 2 & 3 \\
\hline$(3,1)$ & 2 & 0 & 1 & 1 \\
\hline$(3,2)$ & 1 & 0 & 0 & 0 \\
\hline$(3,3)$ & 0 & 0 & 0 & 0 \\
\hline$(3,4)$ & 1 & 0 & 1 & 1 \\
\hline$(3,5)$ & 2 & 0 & 2 & 3 \\
\hline$(4,1)$ & 3 & 0 & 0 & 0 \\
\hline$(4,2)$ & 2 & 0 & 1 & 1 \\
\hline$(4,3)$ & 1 & 0 & 1 & 1 \\
\hline$(4,4)$ & 0 & 0 & 0 & 0 \\
\hline$(4,5)$ & 1 & 0 & 1 & 4 \\
\hline$(5,1)$ & 4 & 0 & 4 & 4 \\
\hline$(5,2)$ & 3 & 0 & 3 & 3 \\
\hline$(5,3)$ & 2 & 0 & 2 & 3 \\
\hline$(5,4)$ & 1 & 0 & 1 & 4 \\
\hline$(5,5)$ & 0 & 0 & 0 & 0 \\
\hline
\end{tabular}

Hence, Q is a weakly multivalued $Z_{(0.5,0.3)}$-contraction. Thus, by Theorem 3, Q is a MWP operator.

Remark 1 . The weakly multivalued $\mathscr{Z}_{(\mathfrak{g}, \mathfrak{r})}$-contraction is the generalization of Popescu notion of $(\mathfrak{g}, \mathfrak{x})$-contractions. By using Definition 3 for some $\mu, \omega \in \mathcal{N}$ and $\mathfrak{g}=0.5$, we have

$$
\mathscr{D}(1, Q 3)=\mathfrak{s} d(1,3)
$$

which implies

$$
\begin{aligned}
& \mathfrak{x} M(1,3)=0.8<\mathscr{H}(\mathbb{Q} 1, \mathbb{Q} 3)=1, \\
& \mathscr{D}(4, \mathbb{Q} 2)=\mathfrak{g} d(4,2),
\end{aligned}
$$

which implies

$$
\begin{aligned}
& \mathfrak{r} M(4,2)=0.8<\mathscr{H}(\mathscr{Q} 4, \mathbb{Q} 2)=1, \\
& \mathscr{D}(5, \mathscr{Q} 1)<\mathfrak{g d}(5,1),
\end{aligned}
$$

which implies

$$
\mathfrak{r} M(5,1)=0.4<\mathscr{H}(\mathscr{Q} 5, Q 1)=4 .
$$

Hence, from equations (44)-(48), it is clear that the notion of $(\mathfrak{g}, \mathfrak{x})$-contraction defined by Popescu [7] is failed for Example 3.
Theorem 4. Let $(\mathcal{N}, d)$ be a complete metric space and $Q: \mathcal{N} \longrightarrow \mathcal{N}$ be a weakly single-valued $\mathscr{Z}_{(\mathfrak{g}, \mathfrak{r})}$-contraction operator. Then, $\mathbb{Q}$ possesses a fixed point. Moreover, if $\mathfrak{Z} \geq 1$ and $L+\mathfrak{r}<1$, then $Q$ possesses a unique fixed point.

Proof. From Theorem 3, Q possesses a fixed point. Suppose that $\mathfrak{G} \geq 1$ and $L+\mathfrak{r}<1$. Assume that $\mathbb{Q}$ possesses two distinct fixed points $\mu$ and $\omega$. Then,

$$
\begin{aligned}
& \mathscr{D}(\omega, \mathbb{Q} \mu)=d(\mu, \omega) \leq \mathfrak{g} d(\mu, \omega) \\
& \Rightarrow \zeta(\mathscr{H}(Q \mu, Q \omega), \mathfrak{r} M(\mu, \omega)) \geq 0 .
\end{aligned}
$$

From (49) and by using $\left(\zeta_{1}\right)$, we have

$$
\begin{gathered}
\zeta(\mathscr{H}(\mathbb{Q} \mu, \mathbb{Q} \omega), \mathfrak{r} M(\mu, \omega))<\mathfrak{r} M(\mu, \omega))-\mathscr{H}(\mathbb{Q} \mu, \mathbb{Q} \omega) \\
=(\mathfrak{r}+L) d(\mu, \omega))-d(\mu, \omega)=(\mathfrak{r}+L-1) d(\mu, \omega)) .
\end{gathered}
$$

We know that $\mathfrak{r}+L-1<0$. Therefore, we get $\zeta(\mathscr{H}(Q \mu, Q \omega), \mathfrak{x} M(\mu, \omega))<0$, which is a contradiction to our assumption. Thus, $Q$ possesses a unique fixed point.

Theorem 5. Let $Q$ be a weakly multivalued $\mathscr{Z}_{(\mathfrak{B}, \mathfrak{r})}$-contraction from $\mathcal{N}$ into $\mathscr{C} \mathscr{B}(\mathcal{N})$. Assume that there exist $\mathfrak{r}, \mathfrak{g} \in(0,1)$ such that

$$
\frac{1}{1+\mathfrak{r}} \mathscr{D}(\mu, \mathcal{Q} \mu) \leq d(\mu, \omega) \leq \frac{1}{1-\mathfrak{G}} \mathscr{D}(\mu, \mathcal{Q} \mu),
$$


which implies

$$
\zeta(\mathscr{H}(Q \mu, Q \omega), \mathfrak{r} M(\mu, \omega)) \geq 0
$$

Then, $\mathbb{Q}$ is a Picard operator.

Proof. Without loss of generality choose Q. Take a real number $\mathfrak{t}$ such that $0<\mathfrak{r}<\mathfrak{t}<\mathfrak{g}$. Let $\mu_{1} \in \mathscr{N}$ and $\mu_{2} \in Q 2 \mu_{1}$ such that $d\left(\mu_{1}, \mu_{2}\right) \leq((1-\mathfrak{t}) /(1-\mathfrak{g})) \mathscr{D}\left(\mu_{1}, Q \mathcal{Q} \mu_{1}\right)$.

Then,

$$
\frac{1}{1+\mathfrak{r}} \mathscr{D}\left(\mu_{1}, \mathscr{Q} \mu_{1}\right) \leq \mathscr{D}\left(\mu_{1}, \mathscr{Q} \mu_{1}\right) \leq d\left(\mu_{1}, \mu_{2}\right) \leq \frac{1}{1-\mathfrak{g}} \mathscr{D}\left(\mu_{1}, \mathscr{Q} \mu_{1}\right) .
$$

By hypothesis, we have

$$
0 \leq \zeta\left(\mathscr{H}\left(Q \mu_{1}, Q \mu_{2}\right), \mathfrak{r} M\left(\mu_{1}, \mu_{2}\right)\right)<\mathfrak{r} M\left(\mu_{1}, \mu_{2}\right)-\mathscr{H}\left(Q \mu_{1}, Q \mu_{2}\right) .
$$

So,

$$
\mathscr{H}\left(Q \mu_{1}, Q \mu_{2}\right)<\mathfrak{r} M\left(\mu_{1}, \mu_{2}\right) \text {. }
$$

Following similar steps as in Theorem 3, we can easily obtain that $d\left(\mu_{2}, \mu_{3}\right)<\mathfrak{r} d\left(\mu_{1}, \mu_{2}\right)$. Therefore, a sequence $\left\{\mu_{\mathbf{n}}\right\}$ can be constructed in $\mathcal{N}$ such that $\mu_{\mathbf{n}+1} \in Q \mu_{\mathbf{n}}$ and $d\left(\mu_{\mathbf{n}+1}, Q \mu_{\mathbf{n}+2}\right)<\mathbf{t} d\left(\mu_{\mathbf{n}}, \mu_{\mathbf{n}+2}\right)$ for all $\mathbf{n} \in \mathbf{N}$

$$
\sum_{\mathbf{n}=1}^{\infty} d\left(\mu_{\mathbf{n}}, \mu_{\mathbf{n}+1}\right)<\sum_{\mathbf{n}=1}^{\infty} \mathbf{t}^{\mathbf{n}-1} d\left(\mu_{1}, \mu_{2}\right)<\infty .
$$

That is, $\left\{\mu_{\mathbf{n}}\right\}$ is a Cauchy sequence. Completeness of $\mathscr{N}$ yields that there is $u \in \mathcal{N}$ so that $\left\{\mu_{\mathrm{n}}\right\}$ converges to $u$.

Since

$$
\begin{aligned}
& d\left(\mu_{\mathbf{n}+p}, \mu_{\mathbf{n}}\right) \leq d\left(\mu_{\mathbf{n}}, \mu_{\mathbf{n}+1}\right)+d\left(\mu_{\mathbf{n}+1}, \mu_{\mathbf{n}+2}\right)+\cdots+d\left(\mu_{\mathbf{n}+p-1}, \mu_{\mathbf{n}+p}\right) \\
& <d\left(\mu_{\mathbf{n}}, \mu_{\mathbf{n}+1}\right)\left(1+\mathfrak{t}+\mathbf{t}^{2}+\mathfrak{t}^{3}+\cdots+\mathbf{t}^{p-1}\right) \\
& \leq \frac{1-\mathbf{t}^{p}}{1-\mathbf{t}} d\left(\mu_{\mathbf{n}}, \mu_{\mathbf{n}+1}\right), \quad \forall \mathbf{n} \geq N, p \geq 1 .
\end{aligned}
$$

By taking $p \longrightarrow \infty$, we obtain

$$
d\left(u, \mu_{\mathbf{n}}\right)<\frac{1}{1-\mathrm{t}} d\left(\mu_{\mathbf{n}}, \mu_{\mathbf{n}+1}\right), \quad \forall \mathbf{n} \geq 1 .
$$

We have

$$
d\left(\mu_{\mathbf{n}}, \mu_{\mathbf{n}+1}\right) \leq \frac{1-\mathfrak{t}}{1-\mathfrak{g}} \mathscr{D}\left(\mu_{\mathbf{n}}, \mathcal{Q} \mu_{\mathbf{n}}\right), \quad \forall \mathbf{n} \in \mathbb{N}
$$

$$
d\left(u, \mu_{\mathbf{n}+1}\right)<\frac{1}{1-\mathfrak{G}} \mathscr{D}\left(\mu_{\mathbf{n}}, \mathscr{Q} \mu_{\mathbf{n}}\right), \quad \forall \mathbf{n} \in \mathbb{N}
$$

Suppose now that there is $N>0$, such that

$$
d\left(u, \mu_{\mathbf{n}}\right)<\frac{1}{1+\mathfrak{r}} \mathscr{D}\left(\mu_{\mathbf{n}}, \mathscr{Q} \mu_{\mathbf{n}}\right), \quad \forall \mathbf{n} \geq 0 .
$$

Therefore,

$$
d\left(\mu_{\mathbf{n}}, \mu_{\mathbf{n}+1}\right) \leq d\left(u, \mu_{\mathbf{n}}\right)+d\left(u, \mu_{\mathbf{n}+1}\right)
$$

$$
<\frac{1}{1+\mathfrak{r}}\left[\mathscr{D}\left(\mu_{\mathbf{n}}, \mathcal{Q} \mu_{\mathbf{n}}\right)+\mathscr{D}\left(\mu_{\mathbf{n}+1}, \mathcal{Q} \mu_{\mathbf{n}+1}\right)\right]
$$

$$
<\frac{1}{1+\mathfrak{r}}\left[d\left(\mu_{\mathbf{n}}, \mu_{\mathbf{n}+1}\right)+\mathfrak{r} d\left(\mu_{\mathbf{n}}, \mu_{\mathbf{n}+1}\right)\right]
$$

implies

$$
d\left(\mu_{\mathbf{n}}, \mu_{\mathbf{n}+1}\right)<\mathscr{D}\left(\mu_{\mathbf{n}}, \mathscr{Q} \mu_{\mathbf{n}}\right),
$$

a contradiction. So, there exists a subsequence $\left\{\mu_{\mathbf{n}(\mathfrak{m})}\right\}$ of $\left\{\mu_{\mathbf{n}}\right\}$ such that

$$
d\left(u, \mu_{\mathbf{n}(\mathfrak{m})}\right) \geq \frac{1}{1+\mathfrak{r}} \mathscr{D}\left(\mu_{\mathbf{n}(\mathfrak{m})}, Q \mathcal{Q} \mu_{\mathbf{n}(\mathfrak{m})}\right), \quad \forall \mathfrak{m} \geq N .
$$

Since

$$
d\left(u, \mu_{\mathbf{n}(\mathfrak{m})}\right) \leq \frac{1}{1-\mathfrak{g}} \mathscr{D}\left(\mu_{\mathbf{n}(\mathfrak{m})}, \mathscr{Q} \mu_{\mathbf{n}(\mathfrak{m})}\right), \quad \forall \mathbf{n} \geq 1
$$

thus, we have

$$
\begin{aligned}
& \frac{1}{1+\mathfrak{r}} \mathscr{D}\left(u, Q \mathcal{Q} \mu_{\mathbf{n}(\mathfrak{m})}\right) \leq \mathscr{D}\left(u, \mathscr{Q} \mu_{\mathbf{n}(\mathfrak{m})}\right) \\
& \quad \leq d\left(u, \mu_{\mathbf{n}(\mathfrak{m})}\right) \leq \frac{1}{1-\mathfrak{g}} \mathscr{D}\left(u, Q \mathcal{Q} \mu_{\mathbf{n}(\mathfrak{m})}\right) .
\end{aligned}
$$

This implies that

$$
\zeta\left(\mathscr{H}\left(Q u, Q \mu_{\mathbf{n}(\mathfrak{m})}\right), \mathfrak{r} M\left(u, \mu_{\mathbf{n}(\mathfrak{m})}\right)\right) \geq 0 .
$$

From $(66),\left(\zeta_{1}\right)$, and $\left(\zeta_{2}\right)$, one obtains 


$$
\begin{aligned}
0 \leq & \lim _{\mathbf{n} \longrightarrow \infty} \sup \left[\zeta\left(\mathscr{H}\left(\mathscr{Q} u, \mathscr{Q} \mu_{\mathbf{n}(\mathfrak{m})}\right), \mathfrak{r} M\left(u, \mu_{\mathbf{n}(\mathfrak{m})}\right)\right)\right] \\
< & \lim _{\mathbf{n} \longrightarrow \infty} \sup \left[\mathfrak{r} M\left(u, \mu_{\mathbf{n}(\mathfrak{m})}\right)-\mathscr{H}\left(\mathscr{Q} u, \mathscr{Q} \mu_{\mathbf{n}(\mathfrak{m})}\right)\right] \\
= & \lim _{\mathbf{n} \longrightarrow \infty} \sup \left[\mathfrak{r} \max \left\{d\left(u, \mu_{\mathbf{n}(\mathfrak{m})}\right), \mathscr{D}(u, \mathscr{Q} u), \mathscr{D}\left(\mu_{\mathbf{n}(\mathfrak{m})}, \mathscr{Q} \mu_{\mathbf{n}(\mathfrak{m})}\right), \frac{1}{2}\left(\mathscr{D}\left(u, \mathscr{Q} \mu_{\mathbf{n}(\mathfrak{m})}\right)+\mathscr{D}\left(\mu_{\mathbf{n}(\mathfrak{m})}, \mathscr{Q} u\right)\right)\right\}\right. \\
& \left.+L \min \left\{d\left(u, \mu_{\mathbf{n}(\mathfrak{m})}\right), \mathscr{D}\left(\mu_{\mathbf{n}(\mathfrak{m})}, \mathscr{Q} u\right)\right\}-\max \left\{\mathscr{D}\left(\mathscr{Q} u, \mathscr{Q} \mu_{\mathbf{n}(\mathfrak{m})}\right), \mathscr{D}\left(\mathscr{Q} \mu_{\mathbf{n}(\mathfrak{m})}, \mathscr{Q} u\right)\right\}\right] \\
= & \lim _{\mathbf{n} \longrightarrow \infty} \sup \left[\mathfrak{r} \max \left\{d\left(u, \mu_{\mathbf{n}(\mathfrak{m})}\right), \mathscr{D}(u, \mathscr{Q} u), \mathscr{D}\left(\mu_{\mathbf{n}(\mathfrak{m})}, \mu_{\mathbf{n}(\mathfrak{m})+1}\right), \frac{1}{2}\left(\mathscr{D}\left(u, \mu_{\mathbf{n}(\mathfrak{m})+1}\right)+\mathscr{D}\left(\mu_{\mathbf{n}(\mathfrak{m})}, \mathscr{Q} u\right)\right)\right\}\right. \\
& \left.+L \min \left\{d\left(u, \mu_{\mathbf{n}(\mathfrak{m})}\right), \mathscr{D}(\mu(\mathfrak{m}), \mathscr{Q} u)\right\}-\max \left\{\mathscr{D}\left(\mathscr{Q} u, \mu_{\mathbf{n}(\mathfrak{m})+1}\right), \mathscr{D}\left(\mu_{\mathbf{n}(\mathfrak{m})+1}, \mathscr{Q} u\right)\right\}\right] \\
= & \sup \left[\mathfrak{r} \max \left\{d(u, u), \mathscr{D}(u, \mathscr{Q} u), \mathscr{D}(u, u), \frac{1}{2}(\mathscr{D}(u, u)+\mathscr{D}(u, \mathscr{Q} u))\right\}\right. \\
& +L \min \{d(u, u), \mathscr{D}(u, \mathscr{Q} u)\}-\max \{\mathscr{D}(\mathscr{Q} u, u), \mathscr{D}(u, \mathscr{Q} u)\}] \\
= & (\mathfrak{r}-1) \mathscr{D}(u, \mathscr{Q} u), \\
= & \sup \left[\mathfrak{r} \max \left\{d(u, u), \frac{D(u, \mathscr{Q} u)}{2}\right\}+L \min \{0, \mathscr{D}(u, \mathscr{Q} u)\}-\max \{\mathscr{D}(\mathscr{Q} u, u), \mathscr{D}(u, \mathscr{Q} u)\}\right] \\
& \sup \{(u, \mathscr{Q} u)+0-\mathscr{D}(u, \mathscr{Q} u)] \\
& \\
&
\end{aligned}
$$

where $\mathfrak{r} \in(0,1)$. Hence,

$$
0 \leq(\mathfrak{x}-1) \mathscr{D}(u, \mathscr{Q} u)<0 .
$$

This contradiction shows that $\mathscr{D}(u, Q u)=0$, that is, $u \in Q u$. Hence, $Q$ is a Picard operator.

Corollary 1. Let $\mathbb{Q}$ be a weakly single-valued $\mathscr{Z}_{(\mathfrak{a}, \mathfrak{r})}$-contraction mapping from $\mathcal{N}$ into $\mathcal{N}$. Assume that there exist $\mathfrak{r}, \mathfrak{s} \in[0,1]$ so that

$$
\frac{1}{1+\mathfrak{r}} \mathscr{D}(\mu, \mathcal{Q} \mu) \leq d(\mu, \omega) \leq \frac{1}{1-\mathfrak{G}} \mathscr{D}(\mu, \mathcal{Q} \mu)
$$

implies

$$
\zeta(\mathscr{H}(Q \mu, Q \omega), \mathfrak{r} M(\mu, \omega)) \geq 0
$$

Then, there exists $u \in \mathcal{N}$ such that $u=Q \mathbf{Q} u$.

Proof. One can easily show that, for every $\mu_{1} \in \mathcal{N}$, the sequence $\left\{\mu_{\mathbf{n}}\right\}$ defined by $\mu_{\mathbf{n}+1}=Q \mu_{\mathbf{n}}$ satisfies the relationship $d\left(\mu_{\mathbf{n}+1}, \mu_{\mathbf{n}+2}\right)<\mathfrak{r} d\left(\mu_{\mathbf{n}}, \mu_{\mathbf{n}+1}\right)$ as done in Theorem 3. Thus, the sequence $\left\{\mu_{\mathrm{n}}\right\}$ is Cauchy, so there is $u \in \mathcal{N}$ such that $\lim _{\mathbf{n} \rightarrow \infty} \mu_{\mathbf{n}}=u$. Following Theorem 5 , we can show that $d\left(u, \mu_{\mathbf{n}}\right) \leq(1 /(1-\mathfrak{r})) d\left(\mu_{\mathbf{n}}, \mu_{\mathbf{n}+1}\right)$ for all $\mathbf{n} \geq 1$ and there exists a subsequence $\left\{\mu_{\mathbf{n}(\mathfrak{m})}\right\}$ of $\left\{\mu_{\mathbf{n}}\right\}$ such that $d\left(u, \mu_{\mathbf{n}(\mathfrak{m})}\right) \geq(1 /(1+\mathfrak{r})) d\left(\mu_{\mathbf{n}(\mathfrak{m})}, \mu_{\mathbf{n}(\mathfrak{m})+1}\right)$ for all $\mathfrak{m} \geq N$. Therefore, we obtain that

$$
\begin{aligned}
0 \leq & \lim _{\mathbf{n} \longrightarrow \infty} \sup \left[\zeta\left(\mathscr{H}\left(\mathscr{Q} u, \mathscr{Q} \mu_{\mathbf{n}(\mathfrak{m})}\right), \mathfrak{r} M\left(u, \mu_{\mathbf{n}(\mathfrak{m})}\right)\right)\right] \\
< & \left.\lim _{\mathbf{n} \longrightarrow \infty} \sup \left[\mathfrak{r} M\left(u, \mu_{\mathbf{n}(\mathfrak{m})}\right)\right)-\mathscr{H}\left(\mathscr{Q} u, \mathscr{Q} \mu_{\mathbf{n}(\mathfrak{m})}\right)\right] \\
= & \sup \left[\mathfrak { r } \operatorname { m a x } \left\{d(u, u), \mathscr{D}(u, \mathscr{Q} u), d(u, u), \frac{1}{2}(d(u, u)\right.\right. \\
& +\mathscr{D}(u, \mathscr{Q} u))\} \\
& +L \min \{d(u, u), \mathscr{D}(u, \mathscr{Q} u)\}-\max \{\mathscr{D}(\mathscr{Q} u, u), \mathscr{D}(u, \mathscr{Q} u)\}] \\
= & (\mathfrak{r}-1) \mathscr{D}(u, \mathscr{Q} u)<0, \quad \because \mathfrak{r} \in(0,1) .
\end{aligned}
$$

We deduce that $\mathscr{D}(u, \mathbb{Q} u)=0$. Thus, we get $u=\mathscr{Q} u$.

2.1. Data Dependence of the Fixed Point Set. In this section, we study data dependence of the fixed point set for weakly multivalued $\mathscr{Z}_{(\mathfrak{g}, \mathfrak{r})}$-contractions.

Theorem 6. Let $(\mathcal{N}, d)$ be a metric space and $\mathbb{Q}_{1}$ and $\mathbb{Q}_{2}$ be two multivalued operators. Assume that

(1) $\mathbb{Q}_{i}$ is a weakly $\mathscr{Z}_{\left(\mathfrak{g}, \mathfrak{r}_{i}\right)}$-contraction for each $i \in\{1,2\}$

(2) There exists a real number $\lambda>0$ such that $\mathscr{H}\left(Q_{1} \mu, Q_{2} \mu\right) \leq \lambda, \forall \mu \in \mathscr{N}$

Then, 
(1) $\operatorname{Fix}\left(\mathbb{Q}_{i}\right) \in \mathbb{C}(\mathcal{N})$ for $i \in\{1,2\}$.

(2) $\mathbb{Q}_{1}$ and $\mathbb{Q}_{2}$ are weakly multivalued operators and

$$
\mathscr{H}\left(\operatorname{Fix}\left(Q_{1}\right), \operatorname{Fix}\left(Q_{2}\right)\right) \leq \frac{\lambda}{1-\max \left\{\mathfrak{r}_{1}, \mathfrak{r}_{2}\right\}} .
$$

Proof. From Theorem 3, $\operatorname{Fix}\left(Q_{i}\right)$ is nonempty for $i \in\{1,2\}$. First of all, we will show that the set of fixed point of a weakly multivalued operator $Q$ is closed. Let $\left\{\mu_{\mathrm{n}}\right\}$ be a sequence in $\operatorname{Fix}(\mathbb{Q})$ such that $\mu_{\mathbf{n}} \longrightarrow u$ as $\mathbf{n} \longrightarrow \infty$. One obtains

$$
\mathscr{D}\left(u, Q \mu_{\mathbf{n}}\right) \leq d\left(u, \mu_{\mathbf{n}}\right) \text {. }
$$

This implies that

$$
\zeta\left(\mathscr{H}\left(Q \mu_{\mathbf{n}}, Q u\right), \mathfrak{r} M\left(\mu_{\mathbf{n}}, u\right)\right) \geq 0 .
$$

Now, from (74), $\left(\zeta_{1}\right)$, and $\left(\zeta_{2}\right)$, we have

$$
\begin{aligned}
0 \leq & \lim _{\mathbf{n} \longrightarrow \infty} \sup \left[\zeta\left(\mathscr{H}\left(\mathscr{Q} u, \mathscr{Q} \mu_{\mathbf{n}}\right), \mathfrak{r} M\left(u, \mu_{\mathbf{n}}\right)\right)\right] \\
< & \lim _{\mathbf{n} \longrightarrow \infty} \sup \left[\mathfrak{r} M\left(u, \mu_{\mathbf{n}}\right)-\mathscr{H}\left(\mathscr{Q} u, \mathscr{Q} \mu_{\mathbf{n}}\right)\right] \\
= & \lim _{\mathbf{n} \longrightarrow \infty} \sup \left[\mathfrak{r} \max \left\{d\left(u, \mu_{\mathbf{n}}\right), \mathscr{D}(u, \mathscr{Q} u), \mathscr{D}\left(\mu_{\mathbf{n}}, \mathscr{Q} \mu_{\mathbf{n}}\right), \frac{1}{2}\left(\mathscr{D}\left(u, \mathscr{Q} \mu_{\mathbf{n}}\right)+\mathscr{D}\left(\mu_{\mathbf{n}}, \mathscr{Q} u\right)\right)\right\}\right. \\
& \left.+L \min \left\{d\left(u, \mu_{\mathbf{n}}\right), \mathscr{D}\left(\mu_{\mathbf{n}}, \mathscr{Q} u\right)\right\}-\max \left\{\mathscr{D}\left(\mathscr{Q} u, \mathscr{Q} \mu_{\mathbf{n}}\right), \mathscr{D}\left(\mathscr{Q} \mu_{\mathbf{n}}, \mathscr{Q} u\right)\right\}\right] \\
= & \lim _{n \longrightarrow \infty} \sup \left[\mathfrak{r} \max \left\{d\left(u, \mu_{\mathbf{n}}\right), \mathscr{D}(u, \mathscr{Q} u), \mathscr{D}\left(\mu_{\mathbf{n}}, \mu_{\mathbf{n}+1}\right), \frac{1}{2}\left(\mathscr{D}\left(u, \mu_{\mathbf{n}+1}\right)+\mathscr{D}\left(\mu_{\mathbf{n}}, \mathscr{Q} u\right)\right)\right\}\right. \\
& \left.+L \min \left\{d\left(u, \mu_{\mathbf{n}}\right), \mathscr{D}\left(\mu_{\mathbf{n}}, \mathscr{Q} u\right)\right\}-\max \left\{\mathscr{D}\left(\mathscr{Q} u, \mu_{\mathbf{n}+1}\right), \mathscr{D}\left(\mu_{\mathbf{n}+1}, \mathscr{Q} u\right)\right\}\right] \\
= & \sup \left[\mathfrak{r} \max \left\{d(u, u), \mathscr{D}(u, \mathscr{Q} u), \mathscr{D}(u, u), \frac{1}{2}(\mathscr{D}(u, u)+\mathscr{D}(u, \mathscr{Q} u))\right\}\right. \\
& +L \min \{d(u, u), \mathscr{D}(u, \mathscr{Q} u)\}-\max \{\mathscr{D}(\mathscr{Q} u, u), \mathscr{D}(u, \mathscr{Q} u)\}] \\
= & (\mathfrak{r}-1) \mathscr{D}(u, \mathscr{Q} u)<0, \quad \because \mathfrak{r} \in[0,1) .
\end{aligned}
$$

Thus, from this contradiction, we deduce that $\mathscr{D}(u, \mathbb{Q} u)=0$. Since $\mathbb{Q} u \in \mathfrak{C}(\mathcal{N})$, we have $u \in \mathbb{Q} u$. Hence, $u \in \operatorname{Fix}(Q)$. Secondly, from Theorem 3, we get that a weakly multivalued $\mathscr{Z}_{(1, \mathfrak{r})}$-contractive operator is a MWP operator. Let $\mathfrak{q}>1$ be a real number and $\mu_{0} \in \operatorname{Fix}\left(\mathscr{Q}_{1}\right)$ be arbitrary. Then, there exists $\mu_{1} \in \mathbb{Q}_{2} \mu_{0}$ such that $d\left(\mu_{0}, \mu_{1}\right) \leq \mathscr{H}\left(\mathbb{Q}_{1} \mu_{0}, \mathscr{Q}_{2} \mu_{0}\right)$. Next, for $\mu_{1} \in \mathbb{Q}_{2} \mu_{0}$, there is $\mu_{2} \in \mathbb{Q}_{2} \mu_{1}$ such that $d\left(\mu_{1}, \mu_{2}\right) \leq \mathscr{H}\left(\mathbb{Q}_{2} \mu_{0}, \mathbb{Q}_{2} \mu_{1}\right)$. Since $\mu_{1} \in \mathbb{Q}_{2} \mu_{0}, \mathscr{D}\left(\mu_{1}, Q_{2} \mu_{0}\right)=0 \leq d\left(\mu_{0}, \mu_{1}\right)$. So, we have

$$
d\left(\mu_{1}, \mu_{2}\right) \leq q \mathscr{H}\left(\mathscr{Q}_{2} \mu_{0}, Q_{2} \mu_{1}\right) \leq \mathfrak{q} \mathfrak{r}_{2} d\left(\mu_{0}, \mu_{1}\right) .
$$

Therefore, in a similar way, we obtain that the sequence of successive approximations for $\mathbb{Q}_{2}$ starting from $\mu_{0}$ fulfills the following assertions:

$$
\begin{aligned}
& \mu_{\mathbf{n}+1} \in \mathbb{Q} \mu_{\mathbf{n}}, \\
& d\left(\mu_{\mathbf{n}}, \mu_{\mathbf{n}+1}\right) \leq\left(q \mathfrak{r}_{2}\right)^{n} d\left(\mu_{0}, \mu_{1}\right), \quad \forall \mathbf{n} \geq 1 .
\end{aligned}
$$

Hence, for all $\mathbf{n} \geq N$ and $p \geq 1$,

$$
\begin{aligned}
& d\left(\mu_{\mathbf{n}+p}, \mu_{\mathbf{n}}\right) \leq d\left(\mu_{\mathbf{n}}, \mu_{\mathbf{n}+1}\right)+d\left(\mu_{\mathbf{n}+1}, \mu_{\mathbf{n}+2}\right)+\cdots+d\left(\mu_{\mathbf{n}+p-1}, \mu_{\mathbf{n}+p}\right) \\
& \leq\left(q \mathfrak{r}_{2}\right)^{\mathbf{n}} d\left(\mu_{0}, \mu_{1}\right)+\left(q \mathfrak{r}_{2}\right)^{\mathbf{n}+1} d\left(\mu_{0}, \mu_{1}\right)+\cdots+\left(q \mathfrak{r}_{2}\right)^{\mathbf{n}+p-1} d\left(\mu_{0}, \mu_{1}\right) \\
& \leq \frac{\left(q \mathfrak{r}_{2}\right)^{\mathbf{n}}}{1-q \mathfrak{r}_{2}} d\left(\mu_{0}, \mu_{1}\right) .
\end{aligned}
$$

Choosing now $1<\mathfrak{q}<\min \left\{\left(1 / \mathfrak{r}_{1}\right),\left(1 / \mathfrak{r}_{2}\right)\right\}$ and letting $\mathbf{n} \longrightarrow \infty$, we obtain that $\left\{\mu_{\mathbf{n}}\right\}$ is a Cauchy sequence in $(\mathcal{N}, d)$. Then, there exists $u \in \mathcal{N}$ such that $\mu_{\mathrm{n}} \longrightarrow u$ as $\mathbf{n} \longrightarrow \infty$. We will prove that $u$ is a fixed point for $\mathbb{Q}_{2}$. Suppose that there exists a positive number $N$ such that $\mathscr{D}\left(u, \mathcal{Q} \mu_{\mathbf{n}}\right)>d\left(u, \mu_{\mathbf{n}}\right) \forall \mathbf{n} \geq N$. Then, $\quad d\left(u, \mu_{\mathbf{n}+1}\right)>d$ $\left(u, \mu_{\mathbf{n}}\right) \forall \mathbf{n} \geq N$. Since $\mu_{\mathbf{n}} \longrightarrow u$ as $\mathbf{n} \longrightarrow \infty$, we get a contradiction. Hence, there exists a subsequence $\left\{\mu_{\mathbf{n}(\mathfrak{m})}\right\}$ such that $\mathscr{D}\left(u, \mathscr{Q}_{2} \mu_{\mathbf{n}(\mathfrak{m})}\right) \leq d\left(u, \mu_{\mathbf{n}(m)} \forall \mathfrak{m}\right) \in \mathbb{N}$. Thus,

$$
\zeta\left(\mathscr{H}\left(\mathscr{Q}_{2} u, Q_{2} \mu_{\mathbf{n}(\mathfrak{m})}\right), \mathfrak{r}_{2} M\left(u, \mu_{\mathbf{n}(\mathfrak{m})}\right)\right) \geq 0 .
$$

Now, from (79), $\left(\zeta_{1}\right)$, and $\left(\zeta_{2}\right)$, we have 


$$
\begin{aligned}
& 0 \leq \lim _{\mathbf{n} \longrightarrow \infty} \sup \left[\zeta\left(\mathscr{H}\left(Q_{2} u, Q_{2} \mu_{\mathbf{n}}\right), \mathfrak{r}_{2} M\left(u, \mu_{\mathbf{n}(\mathrm{m})}\right)\right)\right] \\
& <\lim _{\mathbf{n} \longrightarrow \infty} \sup \left[\mathfrak{r}_{2} M\left(u, \mu_{\mathbf{n}(\mathrm{m})}\right)-\mathscr{H}\left(Q_{2} u, Q_{2} \mu_{\mathbf{n}(\mathrm{m})}\right)\right] \\
& =\lim _{\mathbf{n} \longrightarrow \infty} \sup \left[\mathfrak{r}_{2} \max \left\{d\left(u, \mu_{\mathbf{n}(\mathrm{m})}\right), \mathscr{D}\left(u, Q_{2} u\right), \mathscr{D}\left(\mu_{\mathbf{n}(\mathrm{m})}, \mathscr{Q}_{2} \mu_{\mathbf{n}(\mathrm{m})}\right), \frac{1}{2}\left(\mathscr{D}\left(u, \mathscr{Q}_{2} \mu_{\mathbf{n}(\mathrm{m})}\right)+\mathscr{D}\left(\mu_{\mathbf{n}(\mathrm{m})}, \mathscr{Q}_{2} u\right)\right)\right\}\right. \\
& \left.+L \min \left\{d\left(u, \mu_{\mathbf{n}(\mathrm{m})}\right), \mathscr{D}\left(\mu_{\mathbf{n}(\mathrm{m})}, \mathbb{Q}_{2} u\right)\right\}-\max \left\{\mathscr{D}\left(\mathbb{Q}_{2} u, \mathbb{Q}_{2} \mu_{\mathbf{n}(\mathrm{m})}\right), \mathscr{D}\left(\mathbb{Q}_{2} \mu_{\mathbf{n}(\mathrm{m})}, \mathscr{Q}_{2} u\right)\right\}\right] \\
& =\lim _{n \longrightarrow \infty} \sup \left[\mathfrak{r}_{2} \max \left\{d\left(u, \mu_{\mathbf{n}(\mathrm{m})}\right), \mathscr{D}\left(u, Q_{2} u\right), \mathscr{D}\left(\mu_{\mathbf{n}(\mathrm{m})}, \mu_{\mathbf{n}(\mathrm{m})+1}\right), \frac{1}{2}\left(\mathscr{D}\left(u, \mu_{\mathbf{n}(\mathrm{m})+1}\right)+\mathscr{D}\left(\mu_{\mathbf{n}(\mathrm{m})}, \mathscr{Q}_{2} u\right)\right)\right\}\right. \\
& \left.+L \min \left\{d\left(u, \mu_{\mathbf{n}(\mathrm{m})}\right), \mathscr{D}\left(\mu_{\mathbf{n}(\mathrm{m})}, \mathscr{Q}_{2} u\right)\right\}-\max \left\{\mathscr{D}\left(\mathscr{Q}_{2} u, \mu_{\mathbf{n}(\mathrm{m})+1}\right), \mathscr{D}\left(\mu_{\mathbf{n}(\mathrm{m})+1}, \mathscr{Q}_{2} u\right)\right\}\right] \\
& =\sup \left[\mathfrak{r} \max \left\{d(u, u), \mathscr{D}(u, \mathscr{Q} u), \mathscr{D}(u, u), \frac{1}{2}(\mathscr{D}(u, u)+\mathscr{D}(u, \mathscr{Q} u))\right\}\right. \\
& +L \min \{d(u, u), \mathscr{D}(u, \mathscr{Q} u)\}-\max \{\mathscr{D}(\mathscr{Q} u, u), \mathscr{D}(u, \mathscr{Q} u)\}] \\
& =\sup \left[\mathfrak{r} \max \left\{d(u, u), \frac{\mathscr{D}(u, \mathscr{Q} u)}{2}\right\}\right. \\
& +L \min \{0, \mathscr{D}(u, \mathscr{Q} u)\}-\max \{\mathscr{D}(\mathscr{Q} u, u), \mathscr{D}(u, \mathscr{Q} u)\}] \\
& =\sup [\mathfrak{r} \mathscr{D}(u, \mathscr{Q} u)+0-\mathscr{D}(u, \mathscr{Q} u)] \\
& =(\mathfrak{x}-1) \mathscr{D}(u, Q \mathcal{Q} u,
\end{aligned}
$$

where $\mathfrak{r}_{2} \in(0,1)$. So,

$$
0 \leq\left(\mathfrak{r}_{2}-1\right) \mathscr{D}\left(u, Q_{2} u\right)<0
$$

It is a contradiction. So, we obtain that $\mathscr{D}\left(u, Q_{2} u\right)=0$, that is, $u \in \mathbb{Q}_{2} u$. Hence, $u \in \operatorname{Fix}\left(\mathbb{Q}_{2}\right)$.

By taking $p \longrightarrow \infty$ in (78), we have $d\left(u, \mu_{\mathbf{n}}\right) \leq\left(\left(q \mathfrak{r}_{2}\right)^{\mathbf{n}} /\left(1-q \mathfrak{r}_{2}\right)\right) d\left(\mu_{0}, \mu_{1}\right)$ for each $\mathbf{n} \in N$. Then, for $n:=0$, we get $d\left(\mu_{0}, u\right) \leq\left(1 /\left(1-\mathfrak{q} \mathfrak{r}_{2}\right)\right) d\left(\mu_{0}, \mu_{1}\right) \leq\left(\mathfrak{q} \lambda /\left(1-\mathfrak{q} \mathfrak{r}_{2}\right)\right)$. In a similar way, we get that, for each $u_{0} \in \operatorname{Fix}\left(Q_{2}\right)$, there exists $\mu \in \operatorname{Fix}\left(Q_{1}\right) \quad$ such that $d\left(u_{0}, \mu\right) \leq\left(1 /\left(1-\mathfrak{q} \mathfrak{r}_{1}\right)\right) d\left(u_{0}, u_{1}\right) \leq\left(\mathfrak{q} \lambda /\left(1-\mathfrak{q} \mathfrak{r}_{1}\right)\right)$. Hence,

$$
\mathscr{H}\left(\operatorname{Fix}\left(Q_{1}\right), \operatorname{Fix}\left(Q_{2}\right)\right) \leq \frac{\mathfrak{q} \lambda}{1-\max \left\{\mathfrak{q} \mathfrak{r}_{1}, \mathfrak{q} \mathfrak{r}_{2}\right\}} .
$$

Letting $\mathfrak{q} \longrightarrow 1$ completes the proof. Moreover, we get that $Q_{i}$ is a MWP operator for $i \in\{1,2\}$.

2.2. Strict Fixed Point and Well-Posedness. Now, we prove the well-posedness for a weakly multivalued $\mathscr{Z}_{(\mathfrak{g}, \mathfrak{r})}$-contractive operator with $\mathfrak{g}>1$.

Theorem 7. Let $(\mathcal{N}, d)$ be a complete metric space and Q: $\mathcal{N} \longrightarrow \mathfrak{S}(\mathcal{N})$ be a multivalued operator. Assume that

(i) Q is a weakly multivalued $\mathscr{Z}_{(\mathfrak{B}, \mathfrak{r})}$-contractive operator with $\mathfrak{g} \geq 1$ (ii) $\operatorname{SFix}(Q) \neq \varnothing$

Then,

(a) $\operatorname{Fix}(Q)=\operatorname{SFix}(Q)=\{z\}$

(b) The fixed point problem is well-posed appropriate to $\mathscr{H}$ if $\mathfrak{g}>1$

Proof. (a) We will prove that $\operatorname{Fix}(Q)=z$. We suppose that $u, z \in \operatorname{Fix}(Q)$ with $u \neq z$.

Since

$$
\mathscr{D}(u, Q \mathcal{Q} z)=d(u, z) \leq \mathfrak{S} d(u, z),
$$

one obtains

$$
\zeta(\mathscr{H}(Q u, Q z), \mathfrak{r} M(u, z)) \geq 0 .
$$

By using $\left(\zeta_{1}\right)$, we have

$0 \leq \zeta(\mathscr{H}(Q u, Q z), \mathfrak{r} M(u, z))<\mathfrak{r} M(u, z)-\mathscr{H}(\mathscr{Q} u, Q z)$

Hence, we obtain $\mathscr{H}(\mathbb{Q} u, Q z)<\mathfrak{r} M(u, z)$. We know that $\mathscr{D}(\mathscr{Q} z, u) \leq \mathscr{H}(\mathbb{Q} u, \mathbb{Q} z)$. So,

$$
\begin{aligned}
& \mathscr{D}(\mathscr{Q} z, u)=d(z, u) \leq \mathscr{H}(\mathscr{Q} u, Q z)<\mathfrak{r} M(u, z) \\
& =\mathfrak{r} d(u, z)+L \min \{d(u, z), d(u, Q z)\}=\mathfrak{r} d(u, z) .
\end{aligned}
$$


Thus, $d(z, u)<\mathfrak{r} d(u, z)$, where $\mathfrak{r} \in[0,1)$. It is a contradiction. Hence, we deduce that $u=z$ and $\operatorname{Fix}(Q)=\{z\}$.

(b) Let $\left\{\mu_{\mathbf{n}}\right\} \in \mathcal{N}, \mathbf{n} \in N$, be such that $\mathscr{D}\left(\mu_{\mathbf{n}}, \mathcal{Q} \mu_{\mathbf{n}}\right) \longrightarrow 0$ as $\mathbf{n} \longrightarrow \infty$. We will prove that $d\left(\mu_{\mathbf{n}}, u\right) \longrightarrow 0$ as $\mathbf{n} \longrightarrow \infty$. Arguing by contradiction, we suppose that $d\left(\mu_{\mathbf{n}}, u\right)$ does not converge to 0 . Then, there exist $\varepsilon>0$ and a subsequence $\left\{\mu_{\mathbf{n}(\mathfrak{m})}\right\}$ such that $d\left(\mu_{\mathbf{n}(\mathfrak{m})}, u\right) \geq \varepsilon \forall \mathfrak{m} \in N$. If there exists a subsequence $\left\{\mu_{\mathbf{n}(\mathfrak{m})}\right\}$ of $\left\{\mu_{\mathbf{n}(\mathfrak{m})}\right\}$ such that

$$
\mathscr{D}\left(u, \mathcal{Q} \mu_{\mathbf{n}(\mathfrak{m})}\right) \leq d\left(u, \mu_{\mathbf{n}(\mathfrak{m})}\right)
$$

it implies

$$
\zeta\left(\mathscr{H}\left(\mathbb{Q} u, Q \mu_{\mathbf{n}(\mathfrak{m})}\right), \mathfrak{r} M\left(u, \mu_{\mathbf{n}(\mathfrak{m})}\right)\right) \geq 0 .
$$

By following similar calculations as in Theorem 3, one gets $d\left(u, \mu_{\mathbf{n}(\mathfrak{m})}\right) \longrightarrow 0$ as $\mathbf{n} \longrightarrow \infty$. Hence, we obtain $\varepsilon \leq d\left(\mu_{\mathbf{n}(\mathfrak{m})}, u\right) \longrightarrow 0$ as $\mathbf{n} \longrightarrow \infty$, which is a contradiction to our supposition, that is, $\varepsilon>0$.

Thus, there exists $\mathscr{D}\left(u, \mu_{\mathbf{n}(\mathfrak{m})}\right)>\mathfrak{g} d\left(u, \mu_{\mathbf{n}(\mathfrak{m})}\right)$ for all $\mathfrak{m} \geq \mathfrak{m}_{1}$. We know that $\mathscr{D}\left(\mu_{\mathbf{n}}, Q \mu_{\mathbf{n}}\right) \longrightarrow 0$ as $\mathbf{n} \longrightarrow \infty$, so there exists $\mathscr{D}\left(\mu_{\mathbf{n}(\mathfrak{m})}, \mathscr{Q} \mu_{\mathbf{n}(\mathfrak{m})}\right)<(\mathfrak{g}-1) \mathcal{E}$ for all $\mathfrak{m} \geq \mathfrak{m}_{2}$. Thus,

$$
\begin{gathered}
(\mathfrak{S}-1) \mathcal{E}>\mathscr{D}\left(\mu_{\mathbf{n}(\mathfrak{m})}, \mathscr{Q} \mu_{\mathbf{n}(\mathfrak{m})}\right) \geq \mathscr{D}\left(z, \mathcal{Q} \mu_{\mathbf{n}(\mathfrak{m})}\right) \\
-d\left(u, \mu_{\mathbf{n}(\mathfrak{m})}\right)>\mathfrak{g} d\left(u, \mu_{\mathbf{n}(\mathfrak{m})}\right) \leq(\mathfrak{g}-1) \mathcal{\varepsilon},
\end{gathered}
$$

for all $\mathfrak{m} \geq \mathfrak{m}_{2}$. It is impossible. Therefore, $\mu_{\mathbf{n}} \longrightarrow u$ as $\mathbf{n} \longrightarrow \infty$.

2.3. An Extension of Moț-Petruşel Theorem. Following Reich [19], Moţ and Petruşel initiated the following concepts.

Definition 7. Let $(\mathcal{N}, d)$ be a metric space and $Y \in \mathfrak{P}(\mathscr{N})$. Then, Q: $Y \longrightarrow \mathscr{C} \mathscr{B}(\mathscr{N})$ is called an $(a, b, c)$-KSR multivalued operator if there are $a, b, c \in R_{+}$so that, for $\mu, \omega \in Y$, we have

$$
\begin{aligned}
& \frac{1-b-c}{1+a} \mathscr{D}(\mu, \mathscr{Q} \mu) \leq d(\mu, \omega) \\
& \Rightarrow \mathscr{H}(\mathscr{Q} \mu, \mathscr{Q} \omega) \leq a d(\mu, \omega)+b \mathscr{D}(\mu, \mathbb{Q} \mu)+c \mathscr{D}(\omega, \mathbb{Q} \omega) .
\end{aligned}
$$

Theorem 8. Let $(\mathcal{N}, d)$ be a complete metric space and Q: $\mathcal{N} \longrightarrow \mathscr{C} \mathscr{B}(\mathcal{N})$ be an $(a, b, c)-K S R$ multivalued operator. Then, $\operatorname{Fix}(\mathbb{Q}) \neq \varnothing$. Moreover, $\mathbb{Q}$ is a MWP operator.

Now, we prove the generalization of Theorem 8 in the case $b+c>0$, by using a weakly multivalued $\mathscr{Z}_{(\mathfrak{g}, \mathfrak{r})}$-contractive operator.

Theorem 9. Let $(\mathcal{N}, d)$ be a complete metric space and Q: $\mathscr{N} \longrightarrow \mathscr{C} \mathscr{B}(\mathcal{N})$. Assume that there exist $a, b, c \in R_{+}$with $a+b+c \in(0,1), b+c>0$, so that, for all $\mu, \omega \in \mathcal{N}$, we have

$$
\mathscr{D}(\omega, Q) \leq \frac{a+b+c}{1-b-c} d(\mu, \omega),
$$

which implies

$$
\zeta(\mathscr{H}(Q \mu, Q \omega),(a+b+c) M(\mu, \omega)) \geq 0 .
$$

Then, $\operatorname{Fix}(Q) \neq \varnothing$.

Proof. Let $\mu_{0} \in \mathcal{N}$ and $\mu_{1} \in Q \mu_{0}$. Choose $\mathfrak{r}_{1}$ be a real number such that

$$
0<(a+b+c)<\mathfrak{x}_{1} \leq \frac{a+b+c}{1-b-c}<1 .
$$

One can choose $\mu_{2} \in Q \mu_{1}$, then

$$
\mathscr{D}\left(\mu_{2}, Q \mu_{1}\right)=0 \leq \frac{a+b+c}{1-b-c} d\left(\mu_{2}, \mu_{1}\right),
$$

which implies

$$
\begin{aligned}
0 \leq & \zeta\left(\mathscr{H}\left(Q \mu_{1}, Q \mu_{2}\right),(a+b+c) M\left(\mu_{1}, \mu_{2}\right)\right) \\
& <(a+b+c) M\left(\mu_{1}, \mu_{2}\right)-\mathscr{H}\left(Q \mu_{1}, Q \mu_{2}\right) .
\end{aligned}
$$

That is,

$$
\mathscr{H}\left(Q \mu_{1}, Q \mu_{2}\right)<(a+b+c) M\left(\mu_{1}, \mu_{2}\right) .
$$

Therefore,

$$
\begin{aligned}
& \mathscr{D}\left(\mu_{2}, Q \mu_{2}\right) \leq \mathscr{H}\left(Q \mu_{1}, Q \mu_{2}\right)<(a+b+c) M\left(\mu_{1}, \mu_{2}\right) \\
& =(a+b+c) d\left(\mu_{1}, \mu_{2}\right) .
\end{aligned}
$$

Thus, we obtain

$$
d\left(\mu_{2}, \mu_{3}\right)<(a+b+c) d\left(\mu_{1}, \mu_{2}\right) .
$$

From this way, we can easily construct the sequence $\left\{\mu_{\mathbf{n}}\right\}$ in $\mathcal{N}$ such that $\left\{\mu_{\mathbf{n}+1}\right\} \in Q \mathcal{Q} \mu_{\mathbf{n}}$ and

$$
\begin{aligned}
& d\left(\mu_{\mathbf{n}+1}, \mu_{\mathbf{n}+2}\right)<(a+b+c) d\left(\mu_{\mathbf{n}}, \mu_{\mathbf{n}+1}\right) \\
& <(a+b+c)^{\mathbf{n}-1} d\left(\mu_{1}, \mu_{2}\right) \leq \mathfrak{r}_{1}^{\mathbf{n}-1} d\left(\mu_{1}, \mu_{2}\right) .
\end{aligned}
$$

As $n \longrightarrow \infty$, one obtains

$$
d\left(\mu_{\mathbf{n}+1}, \mu_{\mathbf{n}+2}\right) \longrightarrow 0 .
$$

This shows that $\left\{\mu_{\mathbf{n}}\right\}$ is a Cauchy sequence. Since $\mathcal{N}$ is complete, there is $u \in \mathcal{N}$ so that $\lim _{\mathbf{n} \rightarrow \infty} \mu_{\mathbf{n}}=u$. Now, we suppose that there exists a subsequence $\left\{\mu_{\mathbf{n}(\mathfrak{m})}\right\}$ of $\left\{\mu_{\mathbf{n}}\right\}$ such that

$$
\mathscr{D}\left(u, \mathcal{Q} \mu_{\mathbf{n}(\mathfrak{m})}\right) \leq \frac{a+b+c}{1-b-c} d\left(u, \mu_{\mathbf{n}(\mathfrak{m})}\right),
$$

for all $\mathfrak{m} \in \mathbb{N}$. Assume on the contrary that there is a positive integer $N \in \mathbb{N}$ such that

$$
\mathscr{D}\left(u, Q \mu_{\mathbf{n}}\right)>\frac{a+b+c}{1-b-c} d\left(u, \mu_{\mathbf{n}}\right), \quad \forall \mathbf{n} \in \mathbb{N} .
$$

Then, following similar procedure of Theorem 3, we can show that this contrary assumption leads to the contradiction. Therefore, there exists a subsequence $\left\{\mu_{\mathbf{n}(\mathfrak{m})}\right\}$ of $\left\{\mu_{\mathbf{n}}\right\}$ such that 


$$
\mathscr{D}\left(u, Q \mu_{\mathbf{n}(\mathfrak{m})}\right) \leq \frac{a+b+c}{1-b-c} d\left(u, \mu_{\mathbf{n}(\mathfrak{m})}\right)
$$

for all $\mathfrak{m} \in \mathbb{N}$. This implies that

$$
\zeta\left(\mathscr{H}\left(Q u, Q \mu_{\mathbf{n}(\mathfrak{m})}\right),(a+b+c) M\left(u, \mu_{\mathbf{n}(\mathfrak{m})}\right)\right) \geq 0 .
$$

By solving this inequality, using the definition of a simulation function, we will obtain $\mathscr{D}(u, \mathscr{Q} u)=0$, that is, $u \in Q u$. Hence, $\operatorname{Fix}(Q) \neq \varnothing$.

\section{An Application}

Many authors [20-22] have studied the existence and uniqueness of a solution of functional equations arising in dynamic programming. We make use of Theorem 4 to explore the existence and uniqueness of a solution for a class of functional equations. From now on, $\mathfrak{U}$ and $\mathfrak{V}$ are Banach spaces and $\mathfrak{W} \subset \mathcal{U}, \mathfrak{D} \subset \mathfrak{V}$, and $\mathfrak{R}$ is the set of real numbers. We denote by $\mathbb{B}(\mathfrak{W})$ the set of all bounded real-valued functions on $\mathfrak{W}$. The set $\mathbb{B}(\mathfrak{W})$ equipped with the metric,

$$
d_{\mathbb{B}}(\mathfrak{h}, \mathfrak{m})=\sup _{\mu \in \mathfrak{W}}|\mathfrak{h}(\mu)-\mathfrak{m}(\mu)|, \mathfrak{h}, \mathfrak{m} \in \mathbb{B}(\mathfrak{W}),
$$

is a complete metric space. Viewing $\mathfrak{W}$ and $\mathfrak{D}$ as the state and decision space, respectively, the problem of dynamic programming reduces to the problem of solving the functional equation $\left.\mathfrak{P}(\mu)=\sup _{\omega \in \mathfrak{D}} \mathscr{H}(\mu, \omega, p(t)(\mu, \omega))\right)$, where $\mathrm{t}: \mathfrak{W} \times \mathfrak{D} \longrightarrow \mathfrak{W}$ represents the transformation of the process and $\mathfrak{P}(\mu)$ corresponds to the optimal return function with an initial functional equation:

$$
\mathfrak{P}(\mu)=\sup _{\omega \in \mathfrak{D}}\{g(\mu, \omega)+G(\mu, \omega, p(\mathfrak{t}(\mu, \omega)))\}, \quad \mu \in \mathfrak{W},
$$

where $g: \mathfrak{W} \times \mathfrak{D} \longrightarrow \mathfrak{R}$ and $G: \mathfrak{W} \times \mathfrak{D} \times \mathfrak{R} \longrightarrow \mathfrak{R}$ are bounded functions. Let $Q$ be defined by $\mathbb{Q}(\mathfrak{h}(\mu))=\sup _{\omega \in \mathfrak{D}}\{g(\mu, \omega)+G(\mu, \omega, p(\mathfrak{t}(\mu, \omega)))\}$, where $\mathfrak{h} \in \mathbb{B}(\mathfrak{W})$ and $\mu \in \mathfrak{W}$.

Theorem 10. Suppose that there exist $\mathfrak{x} \in(0,1), \mathfrak{g}>\mathfrak{r}$ such that, for every $(\mu, \omega) \in \mathfrak{W} \times \mathfrak{D}, \mathfrak{h}, \mathfrak{m} \in \mathbb{B}(\mathfrak{W})$ and $\mathfrak{t} \in \mathfrak{W}$, the inequality

$$
|\mathfrak{m}(\mathfrak{t})-\mathscr{Q}(\mathfrak{h}(\mathfrak{t}))| \leq \mathfrak{G}|\mathfrak{m}(\mathfrak{t})-\mathfrak{h}(\mathfrak{t})|
$$

implies

$$
\zeta(|G(\mu, \omega, \mathfrak{h}(\mathfrak{t}))-G(\mu, \omega, \mathfrak{m}(\mathfrak{t}))|, \mathfrak{r} M(\mathfrak{h}(\mathfrak{t}), \mathfrak{m}(\mathfrak{t}))) \geq 0,
$$

where

$$
\begin{aligned}
M(\mathfrak{h}(\mathfrak{t}), \mathfrak{m}(\mathfrak{t}))= & \max \{|\mathfrak{h}(\mathfrak{t})-\mathfrak{m}(\mathfrak{t})|,|\mathfrak{m}(\mathfrak{t})-\mathscr{Q}(\mathfrak{m}(\mathfrak{t}))|, \mid \mathfrak{h}(\mathfrak{t})-\mathscr{Q}(\mathfrak{h}(\mathfrak{t})), \\
& \left.\frac{|\mathfrak{h}(\mathfrak{t})-\mathscr{Q}(\mathfrak{m}(\mathfrak{t}))|+|\mathfrak{m}(\mathfrak{t})-\mathscr{Q}(\mathfrak{h}(\mathfrak{t}))|}{2}\right\} \\
& +\mathscr{L} \min \{|\mathfrak{h}(\mathfrak{t})-\mathfrak{m}(\mathfrak{t})|,|\mathfrak{m}(\mathfrak{t})-\mathscr{Q}(\mathfrak{h}(\mathfrak{t}))|\},
\end{aligned}
$$

with $\mathscr{L}=L \geq 0$. Then, the functional equation (106) possesses a bounded solution. Moreover, if $\mathfrak{g} \geq 1$ and $L+\mathfrak{r}<1$, then such a solution is unique.

Proof. Let $\mathcal{Q}$ be a self-map of $\mathbb{B}(\mathfrak{W})$. Let $\lambda$ be an arbitrary real number and $\mathfrak{h}, \mathfrak{m} \in \mathbb{B}(\mathfrak{W})$. Pick $\mu \in \mathfrak{W}$. We can choose $\omega_{1}, \omega_{2} \in \mathfrak{D}$ so that

$$
\begin{gathered}
\mathbb{Q}(\mathfrak{h}(\mu))<g\left(\mu, \omega_{1}\right)+G\left(\mu, \omega_{1}, \mathfrak{h}\left(\mathfrak{t}_{1}\right)\right)+\lambda, \\
\mathbb{Q}(\mathfrak{m}(\mu))<g\left(\mu, \omega_{2}\right)+G\left(\mu, \omega_{2}, \mathfrak{m}\left(\mathfrak{t}_{2}\right)\right)+\lambda,
\end{gathered}
$$

where $\mathbf{t}_{i}=\mathbf{t}_{i}\left(\mu, \omega_{i}\right), i \in\{1,2\}$.

Using the definition of $\mathcal{Q}$, we obtain

$$
\begin{gathered}
\mathcal{Q}(\mathfrak{h}(\mu)) \geq g\left(\mu, \omega_{2}\right)+G\left(\mu, \omega_{2}, \mathfrak{h}\left(\mathfrak{t}_{2}\right)\right), \\
\mathbb{Q}(\mathfrak{m}(\mu)) \geq g\left(\mu, \omega_{1}\right)+G\left(\mu, \omega_{1}, \mathfrak{m}\left(\mathfrak{t}_{1}\right)\right) .
\end{gathered}
$$

If inequality (107) holds, then
$\zeta(|G(\mu, \omega, \mathfrak{h}(\mathfrak{t}))-G(\mu, \omega, \mathfrak{m}(\mathfrak{t}))|, \mathfrak{r} M(\mathfrak{h}(\mu), \mathfrak{m}(\mu))) \geq 0$

From (114) and $\left(\zeta_{1}\right)$, one writes

$$
\begin{aligned}
0 & \leq \zeta(|G(\mu, \omega, \mathfrak{h}(\mathfrak{t}))-G(\mu, \omega, \mathfrak{m}(\mathfrak{t}))|, \mathfrak{r} M(\mathfrak{h}(\mu), \mathfrak{m}(\mu))) \\
& <\mathfrak{r} M(\mathfrak{h}(\mu), \mathfrak{m}(\mu))-|G(\mu, \omega, \mathfrak{h}(\mathfrak{t}))-G(\mu, \omega, \mathfrak{m}(\mathfrak{t}))| .
\end{aligned}
$$

This yields that

$$
|G(\mu, \omega, \mathfrak{h}(\mathfrak{t}))-G(\mu, \omega, \mathfrak{m}(\mathfrak{t}))|<\mathfrak{r} M(\mathfrak{h}(\mu), \mathfrak{m}(\mu)) .
$$

In view of (111), (114), and (117), we have

$$
\begin{aligned}
& \mathscr{Q}(\mathfrak{h}(\mu))-\mathscr{Q}(\mathfrak{m}(\mu))<G\left(\mu, \omega_{1}, \mathfrak{h}\left(\mathfrak{t}_{1}\right)\right)-G\left(\mu, \omega_{1}, \mathfrak{m}\left(\mathfrak{t}_{1}\right)\right)+\lambda \\
& <\mathfrak{r} M(\mathfrak{h}(\mu), \mathfrak{m}(\mu))+\lambda .
\end{aligned}
$$

Similarly, from (112), (113), and (117), we have 


$$
\mathbb{Q}(\mathfrak{m}(\mu))-\mathbb{Q}(\mathfrak{h}(\mu))<\mathfrak{r} M(\mathfrak{h}(\mu), \mathfrak{m}(\mu))+\lambda .
$$

Thus, from equations (117) and (118), the following,

$$
|\mathscr{Q}(\mathfrak{h}(\mu))-\mathscr{Q}(\mathfrak{m}(\mu))|<\mathfrak{r} M(\mathfrak{h}(\mu), \mathfrak{m}(\mu))+\lambda,
$$

holds for all $\mu \in \mathfrak{W}$ and $\lambda>0$. Hence, we get that

$$
d_{\mathbb{B}}(\mathfrak{m}, \mathbb{Q}(\mathfrak{h})) \leq \mathfrak{s} d_{\mathbb{B}}(\mathfrak{m}, \mathfrak{h})
$$

implies

$$
\zeta\left(d_{\mathbb{B}}(\mathbb{Q}(\mathfrak{h}), \mathbb{Q}(\mathfrak{m})), \mathfrak{r} M(\mathfrak{h}, \mathfrak{m})\right) \geq 0,
$$

where

$$
\begin{aligned}
\mathfrak{r} M(\mathfrak{h}, \mathfrak{m})= & \mathfrak{r} \max \left\{|\mathfrak{h}-\mathfrak{m}|,|\mathfrak{m}-\mathscr{Q}(\mathfrak{m})|,|\mathfrak{h}-\mathscr{Q}(\mathfrak{h})|, \frac{|\mathfrak{h}-\mathscr{Q}(\mathfrak{m})|+|\mathfrak{m}-\mathscr{Q}(\mathfrak{h})|}{2}\right\} \\
& +L \min \{|\mathfrak{h}-\mathfrak{m}|,|\mathfrak{m}-\mathscr{Q}(\mathfrak{h})|\}, \quad \text { where } \mathfrak{r} \mathscr{L}=L \geq 0 .
\end{aligned}
$$

Hence, all assertions of Theorem 4 are fulfilled for the mapping $Q$; therefore, we get the required result.

\section{Data Availability}

No data were used to support this study.

\section{Conflicts of Interest}

The authors declare that there are no conflicts of interest regarding the publication of this manuscript.

\section{Authors' Contributions}

All authors contributed equally to the writing of this paper. All authors read and approved the final manuscript.

\section{References}

[1] S. Banach, "Sur les opérations dans les ensembles abstraits et leur application aux équations intégrales," Fundamenta Mathematicae, vol. 3, pp. 133-181, 1922.

[2] S. Nadler, "Multi-valued contraction mappings," Pacific Journal of Mathematics, vol. 30, no. 2, pp. 475-488, 1969.

[3] H. Covitz and S. B. Nadler, "Multi-valued contraction mappings in generalized metric spaces," Israel Journal of Mathematics, vol. 8, no. 1, pp. 5-11, 1970.

[4] P. Patle, D. Patel, H. Aydi, and S. Radenović, "ON H+Type multivalued contractions and applications in symmetric and probabilistic spaces," Mathematics, vol. 7, no. 2, p. 144, 2019.

[5] A. Petrusel, Operational Inclusions, House of the Book of the Science, Cluj-Napoca, Romania, 2002.

[6] I. A. Rus, "Basic problems of the metric fixed point theory revisited (II)," Studia Universitatis Babeş-Bolyai Mathematica, vol. 36, pp. 81-89, 1991.

[7] O. Popescu, "A new type of contractive multivalued operators," Bulletin des Sciences Mathématiques, vol. 137, no. 1, pp. 30-44, 2013.

[8] T. Kamran and S. Hussain, "Weakly (s, r)-contractive multivalued operators," Rendiconti del Circolo Matematico di Palermo (1952), vol. 64, no. 3, pp. 475-482, 2015.

[9] A. Petrusel and I. A. Rus, "Well-posedness of the fixed point problem for multivalued operators," in Applied Analysis and Differential Equations, O. Cârja and I. I. Vrabie, Eds., pp. 295-306, World Scientific, Singapore, 2007.

[10] A. Petrusel, I. A. Rus, and J. C. Yao, "Well-posedness in the generalized sense of the fixed point problems for multivalued operators," Taiwanese Journal of Mathematics, vol. 11, pp. 903-914, 2007.

[11] G. Moț and A. Petruşel, "Fixed point theory for a new type of contractive multivalued operators," Nonlinear Analysis, vol. 70, pp. 3371-3377, 2009.

[12] F. Khojasteh, S. Shukla, and S. Radenovic, "A new approach to the study of fixed point theory for simulation functions," Filomat, vol. 29, no. 6, pp. 1189-1194, 2015.

[13] E. Ameer, H. Aydi, M. Arshad, and M. De la Sen, "Hybrid cirić type graphic $\Upsilon, \Lambda$-contraction mappings with applications to electric circuit and fractional differential equations," Symmetry, vol. 12, no. 3, p. 467, 2020.

[14] E. Karapinar and F. Khojasteh, "An approach to best proximity points results via simulation functions," Journal of Fixed Point Theory and Applications, vol. 19, pp. 1983-1995, 2017.

[15] H. Aydi, H. Lakzian, Z. D. Mitrović, and S. Radenović, "Best proximity points of MT-cyclic contractions with property UC," Numerical Functional Analysis and Optimization, vol. 41, no. 7, pp. 871-882, 2020.

[16] H. Argoubi, B. Samet, and C. Vetro, "Nonlinear contractions involving simulation functions in a metric space with a partial order," Journal of Nonlinear Sciences and Applications, vol. 8, no. 6, pp. 1082-1094, 2015.

[17] A.-F. Roldán-López-de-Hierro, E. Karapınar, C. RoldánLópez-de-Hierro, and J. Martínez-Moreno, "Coincidence point theorems on metric spaces via simulation functions," Journal of Computational and Applied Mathematics, vol. 275, pp. 345-355, 2015.

[18] S. Radenovic and S. Chandok, "Simulation type functions and coincidence points," Filomat, vol. 32, no. 1, pp. 141-147, 2018.

[19] S. Reich, "Fixed points of contractive functions," Bollettino dell'Unione Matematica Italiana, vol. 5, pp. 22-42, 1972.

[20] E. Ameer, H. Aydi, M. Arshad, H. Alsamir, and M. Noorani, "Hybrid multivalued type contraction mappings in $\alpha \mathrm{k}-$ complete partial b-metric spaces and applications," Symmetry, vol. 11, no. 1, p. 86, 2019.

[21] Z. Liu, R. P. Agarwal, and S. M. Kang, "On solvability of functional equations and system of functional equations arising in dynamic programming," Journal of Mathematical Analysis and Applications, vol. 297, no. 1, pp. 111-130, 2004.

[22] Z. Liu, L. Wang, H.-K. Kim, and S.-M. Kang, "Common fixed point theorems for contractive type mappings and their applications in dynamic programming," Bulletin of the Korean Mathematical Society, vol. 45, no. 3, pp. 573-585, 2008. 